\title{
直接三氟甲硫基化反应研究进展
}

\author{
张 柯 ${ }^{a}$ 徐修华 $b \quad$ 卿凤悀*, $a, b$ \\ ( ${ }^{a}$ 东华大学化学化工与生物工程学院 上海 201620) \\ ${ }^{b}$ 中国科学院上海有机化学研究所 有机氟化学重点实验室 上海 200032)
}

\begin{abstract}
摘要 三氟甲硫基由于具有较强的吸电子性和极高的亲脂性，使得含三氟甲硫基的化合物在医药、农药以及材料等领 域发挥着重要的作用. 从有机氟化学的发展历程来看, 三氟甲硫基化反应是继三氟甲基化反应后的又一重要研究课题, 受到化学家的广泛关注. 重点论述了近 5 年来国内外学者在直接三氟甲硫基化反应的研究成果, 并讨论了三氟甲硫基 化反应所面临的一些挑战.
\end{abstract}

关键词 三氟甲硫基化; 有机氟化学; 氧化偶联

\section{Recent Advances of Direct Trifluoromethylthiolation}

\author{
Zhang, $\mathrm{Ke}^{a} \quad \mathrm{Xu}$, Xiuhua $^{b} \quad$ Qing, Fengling*,a,b \\ $\left({ }^{a}\right.$ College of Chemistry, Chemical Engineering and Biotechology, Donghua University, Shanghai 201620) \\ ${ }^{b}$ Key laboratory of Organofluorine Chemistry, Shanghai Institute of Organic Chemistry, \\ Chinese Academy of Sciences, Shanghai 200032)
}

\begin{abstract}
Compounds containing trifluoromethylthio group $\left(\mathrm{SCF}_{3}\right)$ play an important role in pharmaceuticals, agrochemicals and materials due to its strong electron-withdrawing effect and extremely high lipophilicity. Very recently, trifluoromethylthiolation has received a great attention. In this review, the new progress of direct trifluoromethylthiolation is described. Furthermore, the synthetic challenge for trifluoromethylthiolation is also discussed.

Keywords trifluoromethylthiolation; organofluorine chemistry; oxidative coupling
\end{abstract}

2012 年, 本文的作者之一卿风领在本刊发表题为 “从三氟甲基化反应的近年进展看有机氟化学的发展趋 势” 的综述文章中提出, 三氟甲硫基化反应将在未来引 起更多的关注 ${ }^{[1]}$. 正如所预期的那样, 近两年来, 有关 直接三氟甲硫基化反应的论文比过去的总和还多. 众所 周知, 三氟甲硫基 $\left(\mathrm{SCF}_{3}\right)$ 具有强吸电子性和高亲脂性 $\left(\pi_{\mathrm{R}}=1.44\right)^{[2]}$, 而亲脂性的增强有利于提高有机分子的 渗透作用和吸收率, 因此三氟甲硫醚化合物在医药、农 药、材料领域都有着重大的应用前景 ${ }^{[3]}$, 在有机合成上 也具有重要的应用价值 ${ }^{[3 \mathrm{~h}, 3 \mathrm{i}]}$. 目前具有重要生物活性的 含三氟甲硫基的分子主要包括抗球虫药物妥曲珠利 (Toltrazuril)、食欲减退药物替氟雷司(Tiflorex)、抗高血 压药氯沙坦(Losartan analogue) 等 ${ }^{[3 i]}$.

从三氟甲硫基化反应的发展历史可看出, 在 20 世<smiles>CCNC(C)Cc1cccc(C(F)(F)F)c1</smiles>

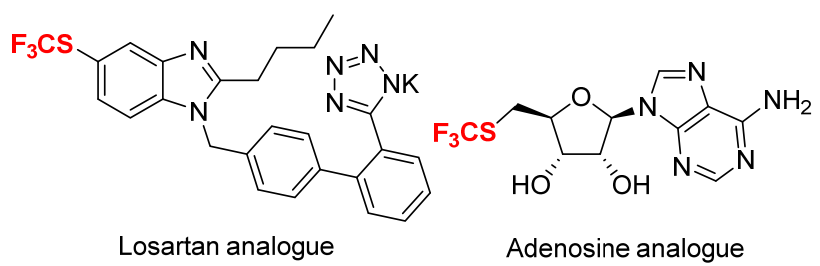

图 1 含三氟甲硫基的代表药物

Figure 1 Typical drugs containing $\mathrm{SCF}_{3}$

* E-mail: flq@mail.sioc.ac.cn

Received January 14, 2015; revised February 9, 2015; published online February 12, 2015.

Project supported by the National Natural Science Foundation of China (Nos. 21421002, 21332010, 21272036) and the National Basic Research Program of China (No. 2012CB21600).

国家自然科学基金(Nos. 21421002, 21332010, 21272036)和国家重点基础研究发展计划(No. 2012CB21600)资助项目. 
纪, 三氟甲硫基的引入方法大部分属于间接方法, 包括 氟卤交换的氟化法 ${ }^{[4]}$ 以及对硫原子的三氟甲基化方法 ${ }^{[5]}$. 然而这些方法都需要预先制备前体, 而且直接氟化法的 反应条件比较苛刻. 进入 21 世纪后, 直接的三氟甲硫基 化反应成为近年来的研究热点. 本文将依据直接三氟甲 硫基化反应的类型, 分别归纳国内外学者在该领域的代 表性工作.

\section{1 自由基三氟甲硫基化}

最早的自由基型三氟甲硫基化反应可以追溯到 1961 年 Harris 发现的三氟甲硫醇 $\left(\mathrm{CF}_{3} \mathrm{SH}\right)$ 对氟烯烃的加 成反应 ${ }^{[6 a]}$. 该反应是在 $X$ 射线或紫外线照射下生成三氟 甲硫自由基然后对氟烯烃进行加成. 1962 年, 他又完成 了三氟甲硫氯 $\left(\mathrm{CF}_{3} \mathrm{SCl}\right)$ 对氟烯烃的加成 ${ }^{[6 b]} .1966$ 年, Harris 又发展了 $\mathrm{CF}_{3} \mathrm{SCl}$ 对烷烃的三氟甲硫基化 ${ }^{[6 \mathrm{cc}]}$. 反应 中产生的烷基自由基优先进攻三氟甲硫氯的硫原子而 不是氯原子, 特别是一级碳自由基的进攻几乎全部发生 在硫原子上, 氯化产物很少(Scheme 1).

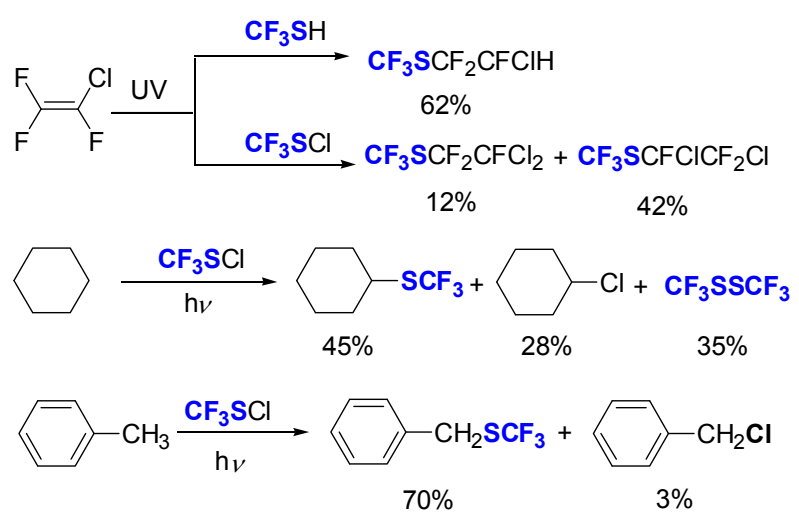

图式 1 氟烯烃和烷烃的自由基三氟甲硫基化反应

Scheme 1 Radical trifluoromethylthiolation of fluoroolefins and alkanes

另一例涉及到三氟甲硫基自由基的反应是 $\mathrm{CF}_{3} \mathrm{SCl}$ 与三甲基硅基烯醇醚之间的反应 ${ }^{[7]}$, 气质联用仪 (GC-MS) 检测到少量的双三氟甲基二硫醚 $\left(\mathrm{CF}_{3} \mathrm{SSCF}_{3}\right)$, 这符合作者所设想的自由基机理, 也解释了各种不同的 副产物的生成(Eq. 1).
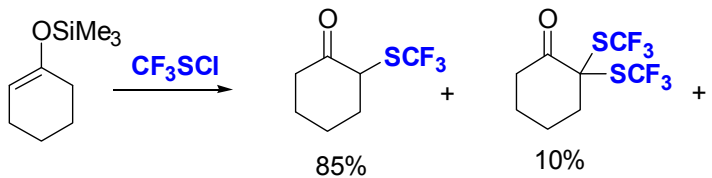<smiles>O=C1C(C(F)(F)F)CCCC1S(F)(F)F</smiles>

双三氟甲基二硫醚在辐射或光照的作用下也能产 生三氟甲硫基自由基, 然后与一些烯烃加成生成双三氟 甲硫基化产物 ${ }^{[8]} .2014$ 年, 中国科学技术大学的王细胜 课题组 ${ }^{[9]}$ 发展了银参与的活化烯烃的芳基三氟甲硫基双 官能团化反应. 在经过一系列的机理实验探索后, 他们 认为反应很可能涉及到三氟甲硫自由基. 三氟甲硫自由 基产生有两种可能途径: 其一，三氟甲硫银 $\left(\mathrm{AgSCF}_{3}\right)$ 在 过硫酸钾的作用下氧化成二价银, 继而产生出三氟甲硫 自由基; 其二, 体系中现场产生的 $\mathrm{CF}_{3} \mathrm{SSCF}_{3}$ 在银盐的 作用下也可能产生三氟甲硫自由基, 然后它对活化了的 双键进行加成得到的碳自由基，随后被分子内苯环捕获 成环, 脱氢得到最终产物(Scheme 2).

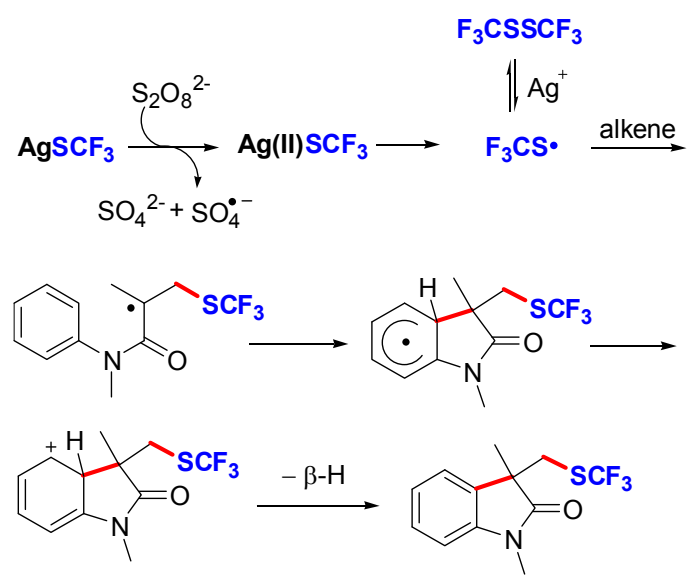

图式 2 银参与的活化烯烃的自由基芳基三氟甲硫基化反应 的可能机理

Scheme 2 Proposed mechanism for silver-mediated radical aryltrifluoromethylthiolaton of activated alkenes

兰州大学基础医学院的王锐、许兆青等 ${ }^{[10]}$ 也报道了 $\mathrm{AgSCF}_{3}$ 作为三氟甲硫基源，醋酸铜催化下的未活化烯 烃的氧一三氟甲硫基化反应. 尽管，该反应的机理并不 明确，但是他们根据以往的报道和相关实验推测: 一方 面, $\mathrm{AgSCF}_{3}$ 经氧化产生三氟甲硫自由基; 另一方面, 肜 在铜的催化下产生氧自由基, 然后进攻烯烃生成碳自由 基，最后和三氟甲硫自由基反应，从而得到烯烃双官能 化的产物(Scheme 3).

\section{2 亲电三氟甲硫基化}

$\mathrm{CF}_{3} \mathrm{SCl}$ 不仅可在光照的条件下产生三氟甲硫基自 由基，在更多的情况下，它还作为一种亲电的三氟甲硫 基化试剂. 在 20 世纪 60 年代至 90 年代之间, $\mathrm{CF}_{3} \mathrm{SCl}$ 与 众多的富电子的底物的亲电反应被报道的很多, 其中包 括有机金属试剂 ${ }^{[1]}$ 、富电子的芳烃 ${ }^{[12]}$ 、杂环芳烃 ${ }^{[13]}$ 、活 泼亚甲基类化合物 ${ }^{[14]}$ 、烯烃 ${ }^{[15]}$ 、烯胺 ${ }^{[16]}$ 等(Scheme 4). 


$$
2 \mathrm{AgSCF}_{3} \underset{-\mathrm{Ag}^{0}}{\longrightarrow} \mathrm{Ag}\left(\mathrm{SCF}_{3}\right)_{2} \longrightarrow \mathrm{F}_{3} \mathrm{CSSCF}_{3} \longrightarrow \mathrm{F}_{3} \mathrm{CS}
$$<smiles>C=CC1=C(c2cccc(C3=NOC(C)C3)c2)CC(c2cccc(C(C)(C)C)c2)=NO1</smiles>

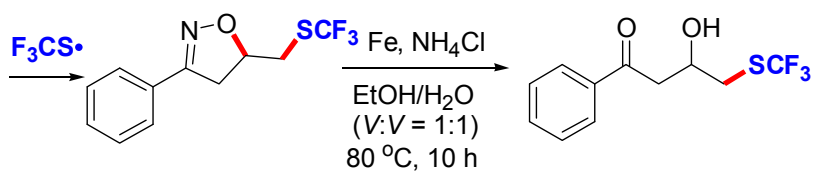

图式 3 铜催化的未活化烯烃的分子内的氧一三氟甲硫基化反 应的可能机理

Scheme 3 Proposed mechanism for copper-catalyzed intramolecular oxytrifluoromethylthiolation of unactivated alkenes

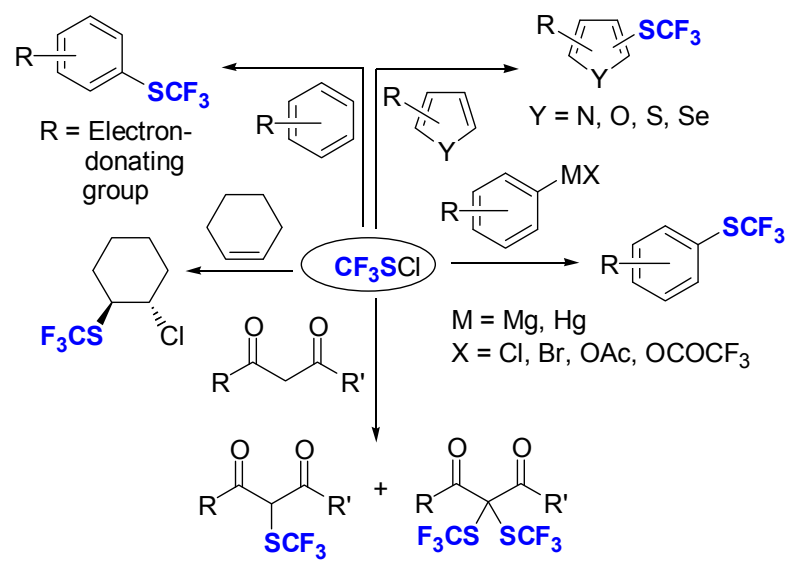

图式 $4 \mathrm{CF}_{3} \mathrm{SCl}$ 与各种亲核底物之间的三氟甲硫基化反应

Scheme 4 Trifluoromethylthiolation of various nucleophilic substrates with $\mathrm{CF}_{3} \mathrm{SCl}$

和 $\mathrm{CF}_{3} \mathrm{SCl}$ 相比, $\mathrm{CF}_{3} \mathrm{SSCF}_{3}$ 作为亲电的三氟甲硫基 化试剂的应用并不多，它能与烷基或芳基钠、锂试剂反 应生成一些简单的三氟甲硫醚产物 ${ }^{[17]}$ (Scheme 5).
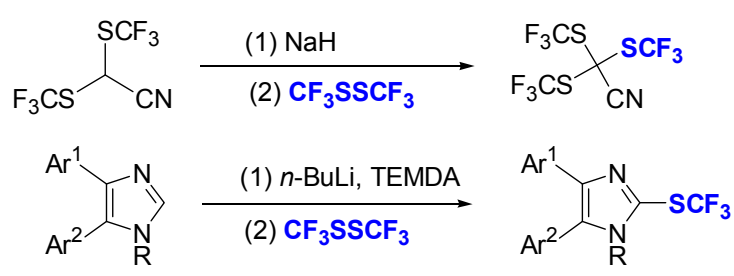<smiles>O=C(Nc1nc(C(F)(F)F)cs1)C(F)(F)C(F)(F)C(F)(F)C(F)(F)C(F)(F)C(F)(F)C(F)(F)C(F)(F)F</smiles>

图式 $5 \mathrm{CF}_{3} \mathrm{SSCF}_{3}$ 与有机钠、锂化合物之间的三氟甲硫基化 反应

Scheme 5 Trifluoromethylthiolation of organolithium and organosodium compounds with $\mathrm{CF}_{3} \mathrm{SSCF}_{3}$
2012 年, Daugulis 课题组 ${ }^{[18]}$ 利用该试剂发展了一类 醋酸铜催化的 8-氨基喹啉芳基苯甲酸酰胺碳-氢 $(\mathrm{C}-\mathrm{H})$ 键直接三氟甲硫基化反应. 这是首例导向基团诱导的芳 烃 $\mathrm{C}-\mathrm{H}$ 键的直接三氟甲硫基化反应(Eq. 2). 该反应具 有良好的普适性，芳环上无论带有拉电子基团还是供电 子基团时，都有较好的收率.

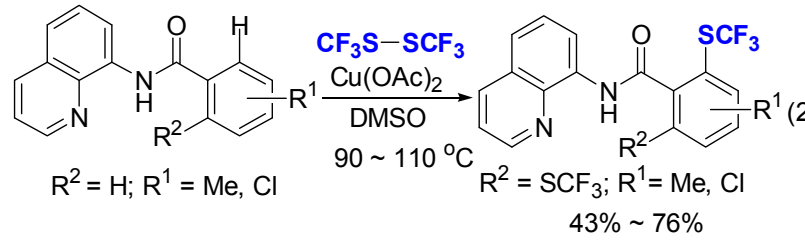

然而，这些早期的亲电型三氟甲硫基化试剂，都存 在着低沸点、高毒性、难操作等缺陷，限制了这些试剂 的发展与使用. 尤其是 $\mathrm{CF}_{3} \mathrm{SSCF}_{3}$, 属于高毒性的气体, 市场上禁止销售. 因此发展新型的亲电的三氟甲硫基化 试剂也成为近年来的热点课题之一.

2008 年, Billard 和 Langlois 等 ${ }^{[19]}$ 通过三氟甲基三甲 基硅烷 $\left(\mathrm{CF}_{3} \mathrm{TMS}\right) 、$ 二氨基三氟化硫(DAST)和苯胺反 应，合成了一类新型的亲电三氟甲硫基化试剂 $N$-三氟 甲硫基苯胺 $\left[\mathrm{PhN}(\mathrm{R}) \mathrm{SCF}_{3}\right]$. 该试剂在路易斯酸的活化 下，能与一系列的亲核试剂反应，包括烯烃 ${ }^{[20] 、}$ 炔 烃 ${ }^{[20,21]}$ 、富电子芳烃 ${ }^{[22]}$ 、有机金属试剂 ${ }^{[23]}$ 以及胺 ${ }^{[24]}$ 等 (Scheme 6), 得到相应的三氟甲硫醚产物. 这个亲电三 氟甲硫基化试剂的发现很好的取代了 $\mathrm{CF}_{3} \mathrm{SCl}$, 在合成 上具有重大的应用价值. 关于该类试剂的拓展应用，目 前依然处于发展之中.

2012 年, 我们课题组 ${ }^{[25]}$ 也利用该试剂实现了路易 斯酸活化的亲电三氟甲硫基化-串联环化反应. 有趣的 是，在对甲苯磺酸的活化下，产物以串联环化产物为主， 而在三氟化嗍乙醚的活化下，主要产物则为 2-三氟甲硫 基取代的吲哚衍生物, 个别产物的选择性可高达 $30 ： 1$. 2013 年, 我们 ${ }^{[26]}$ 又利用该试剂实现了乙酰氯 $\left(\mathrm{CH}_{3} \mathrm{COCl}\right)$ 活化下的烯丙基硅烷的亲电三氟甲硫基化反应. 该反应 无需过渡金属参与、条件温和、具有良好的官能团兼容 性和底物适用性，为烯丙基三氟甲基硫醚的合成提供了 一种有效的合成方法(Scheme 7).

复旦大学的吴劼课题组还利用该试剂在路易斯酸 氯化铋 $\left(\mathrm{BiCl}_{3}\right)$ 的活化下合成了一系列的三氟甲硫基取 代的杂环化合物, 包括苯并 $[1,2]$ 噻嗪 1,1-二氧化物 ${ }^{[27 a] 、}$ 吲哚 ${ }^{[27 b]}$ 、苯并呋喃以及苯并噻吩 ${ }^{[27 c]}$ 等衍生物(Scheme 8). 江西师范大学的丁秋平、陈知远等分别用类似的方 法合成了三氟甲硫基取代的异香豆素衍生物 ${ }^{[27 \mathrm{~d}]}$ 和苯并 富烯烃衍生物 ${ }^{[27 e]}$.

2014 年, Billard 课题组 ${ }^{[28]}$ 在研究 $\mathrm{PhN}(\mathrm{R}) \mathrm{SCF}_{3}$ 与苯 


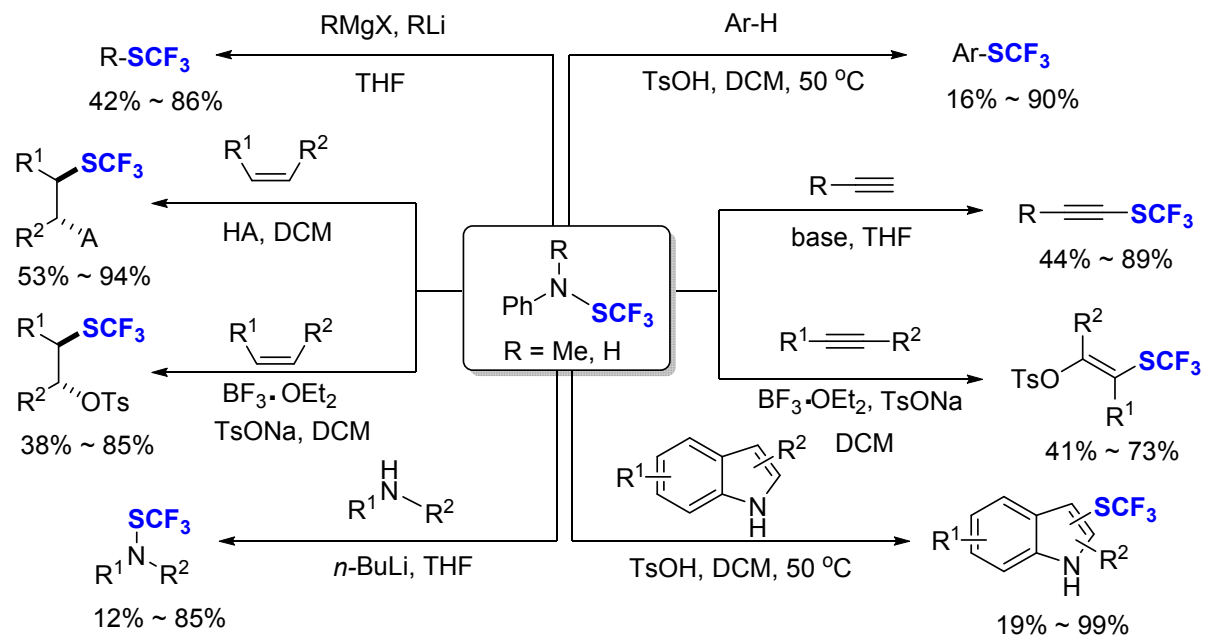

图式 $6 \mathrm{~N}$-三氟甲硫基苯胺与各种亲核体之间的三氟甲硫基化反应

Scheme 6 Trifluoromethylthiolation of various nucleophilic substrates with trifluoromethanesulfenamide

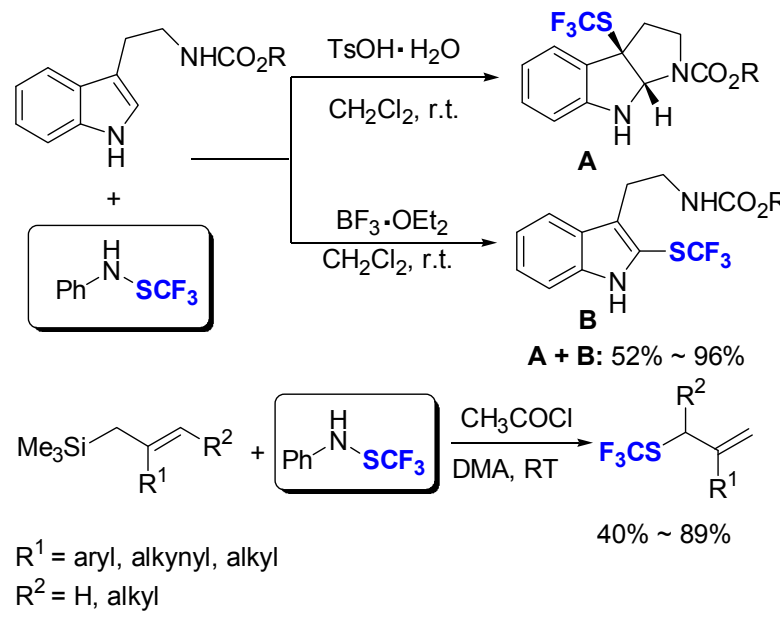

图式 $7 \mathrm{~N}$-三氟甲硫基苯胺与色胺衍生物和烯丙基硅烷之间 的三氟甲硫基化反应

Scheme 7 Trifluoromethylthiolation of tryptamine derivatives and allysilanes with trifluoromethanesulfenamide

乙酮衍生物的反应时发现该试剂的反应活性较弱, 而 $N$-甲基- $N$-三氟甲硫基-4-甲基苯磺酰胺 [ $\mathrm{TsN}(\mathrm{Me}) \mathrm{SCF}_{3}$ ] 则表现出更强的亲电性, 能与各种羰基化合物反应生成 $\alpha$-三氟甲硫基单取代或双取代的羰基化合物. 接着该课 题组 ${ }^{[29]}$ 又用该试剂完成了对杂环化合物的选择性三氟 甲硫基化反应. 该反应首先需要加入强碱夺取底物的氢 原子生成有机金属化合物, 然后与亲电的三氟甲硫基化 试剂反应, 因此对一些对碱敏感的底物不适用(Scheme 9).

$\mathrm{N}$-三氟甲硫基邻苯二甲酰亚胺是另一类重要的亲 电三氟甲硫基化试剂, 在 2000 年, Munavalli 等 ${ }^{\left[{ }^{[0]}\right.}$ 用邻 苯二甲酰亚胺钾盐与 $\mathrm{CF}_{3} \mathrm{SCl}$ 反应制备, 并简单考察了 它作为亲电试剂与亲核试剂的反应, 以乙腈为溶剂, 在 不加任何活化剂的情况下, 室温下与烯胺反应能得到

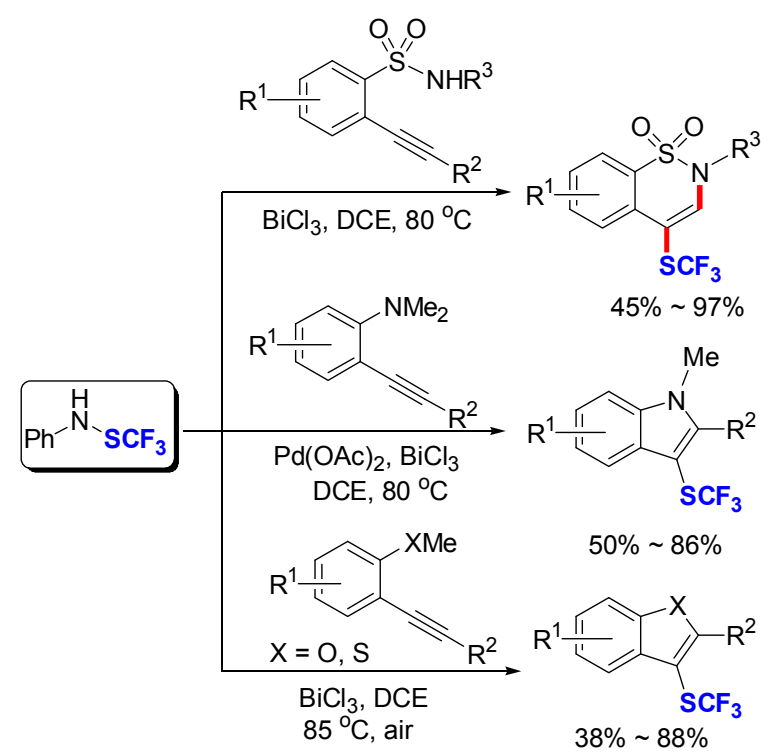

图式 8 合成三氟甲硫基取代的杂环化合物

Scheme 8 Synthesis of $\mathrm{CF}_{3} \mathrm{~S}$-heterocycles with trifluoromethanesulfenamide

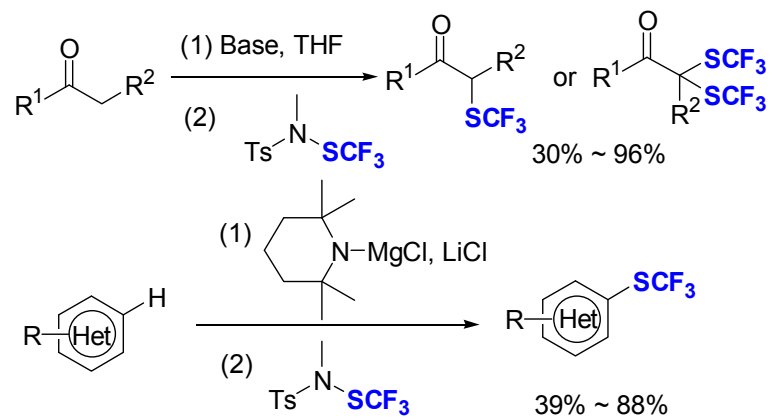

图式 $9\left[\mathrm{TsN}(\mathrm{Me}) \mathrm{SCF}_{3}\right]$ 与羰基化合物、杂环芳烃的亲电三氟 甲硫基化反应

Scheme 9 Trifluoromethylthiolation of carbonyl compounds and deprotonated heteroarenes with $N$-methyl- $N$-tosyl trifluoromethanesulfenamide 
12\%左右的三氟甲硫基化产物，而与活泼亚甲基类化合 物则没有发现相应的产物(Scheme 10).<smiles>CC(F)([NH2+]N1C(=O)c2ccccc2C1=O)C(F)(F)F</smiles><smiles>O=C1c2ccccc2C(=O)N1C(F)(F)F</smiles><smiles>C1=C(N2CCCC2)CCC1</smiles><smiles>CN(C)C</smiles><smiles>O=C1CCCCC1C1CCCCC1C(F)(F)F</smiles>

$n=0,13 \%$ $n=1,12 \%$

图式 $10 \mathrm{~N}$-三氟甲硫基邻苯二甲酰亚胺的合成及其对烯胺的 首例亲电三氟甲硫基化反应

Scheme 10 Synthesis of $N$-(trifluoromethylthio)phthalimide and the first electrophilic trifluoromethylthiolation of enamines

2013 年, Rueping 课题组 ${ }^{[31]}$ 报道了该试剂对 $\beta$-酮酯 的不对称三氟甲硫基化. 在奎宁丁(quinidine)催化下, 该反应不仅产率较高，对映选择性介于 $85 \%$ ～ $99 \%$ ee之 间, 而使用催化量的奎宁 (quinine), 产物的构型则发生 反转, 对映选择性也高达 $96 \%$ ee. 接着, 该课题组 ${ }^{[32]}$ 还 完成了对 2-吲哚酮类衍生物的 $\alpha$ 位不对称三氟甲硫基 化. 在金鸡纳碱手性催化剂(DHQD) ${ }_{2} \operatorname{Pyr}$ (氢化奎宁-2,5二苯基-4,6-嘧啶二甲醚)的催化下，对映应选择性介于 84\% 95\% ee 之间，产率良好到优秀(Scheme 11).

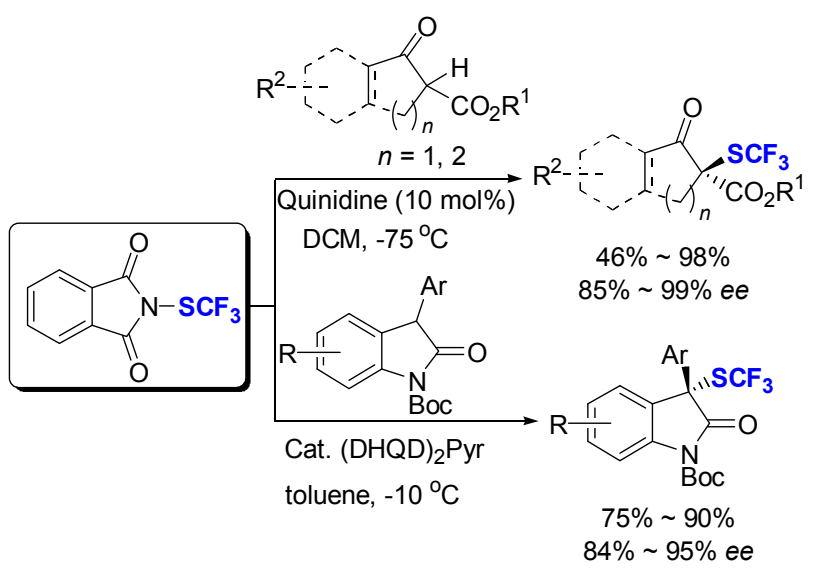

图式 $11 N$-三氟甲硫基邻苯二甲酰亚胺对 $\beta$-酮酯及 2-吲哚酮 类衍生物的不对称三氟甲硫基化反应

Scheme 11 Asymmetric trifluoromethylthiolation of $\beta$-keto esters and oxindoles with $N$-(trifluoromethylthio)phthalimide

2014 年, 该课题组 ${ }^{[33]}$ 也利用该试剂在催化量的铜 盐和 2-2'联吡啶(bpy)的条件下实现了对芳基硼酸包括乙 烯基硼酸和末端炔烃的三氟甲硫基化(Scheme 12).

最近, Rueping 课题组 ${ }^{[34]}$ 报道了该试剂与胺和硫醇 等传统的亲核试剂的反应. 对于伯胺和硫醇等底物, 以 三氟甲苯为溶剂, 加热至 $80{ }^{\circ} \mathrm{C}$ 很容易与 $N$-三氟甲硫基

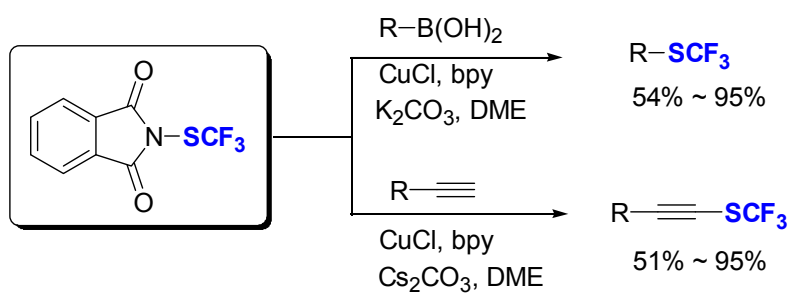

图式 12 铜催化的 $N$-三氟甲硫基邻苯二甲酰亚胺对嗍酸及炔 烃的三氟甲硫基化反应

Scheme $12 \mathrm{Cu}$-catalyzed trifluoromethylthiolation of boronic acids and alkynes with $N$-(trifluoromethylthio)phthalimide

邻苯二甲酰亚胺反应生成相应的取代产物, 对于反应性 稍差的仲胺, 则需要将其制备成相应的锂盐然后再与该 试剂反应(Scheme 13).

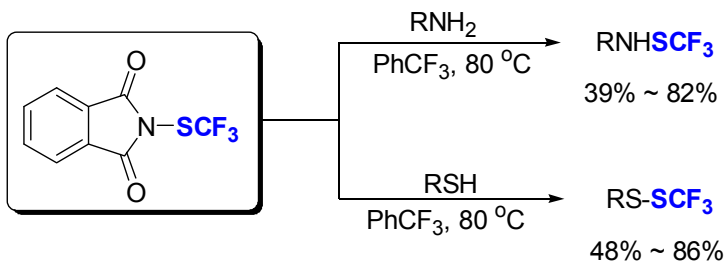

图式 $13 \mathrm{~N}$-三氟甲硫基邻苯二甲酰亚胺与胺及硫醇的亲电三 氟甲硫基化反应

Scheme 13 Electrophilic trifluoromethylthiolation of amines and thiols with $N$-(trifluoromethylthio)phthalimide

2014 年, 中国科学院上海有机化学研究所的沈其 龙课题组 ${ }^{[35]}$ 合成了基于糖精分子设计的新型亲电三氟 甲硫基化试剂—— $N$-三氟甲硫基邻苯甲酰磺酰亚胺. 此 试剂可以由廉价易得的糖精通过两步简单的反应制得， 与 $N$-三氟甲硫基邻苯二甲酰亚胺相比，它具有更好的 反应性，可以在温和的条件下与众多亲核试剂发生反 应，如醇、硫醇、胺、富电子的芳烃、醛、酮以及末端 炔烃等都能以良好的产率得到相应的三氟甲硫基化产 物(Scheme 14), 是向小分子中引入三氟甲硫基的非常 实用的试剂之一.

最近, 中国科学院大连化学物理研究所的李兴伟课 题组 ${ }^{[36]}$ 利用该试剂, 对芳烃以及杂环芳烃的 $\mathrm{C}-\mathrm{H}$ 键直 接活化三氟甲硫基化做了细致的研究工作. 对于杂环芳 烃, 主要是一些吲哚衍生物, 在氯化铁 $\left(\mathrm{FeCl}_{3}\right)$ 的催化下, 能以良好到优秀的收率得到 3-取代的三氟甲硫基取代 的吲哚衍生物. 而对于芳烃, 主要是针对一些富电子的 苯环, 在 $\mathrm{FeCl}_{3}$ 和六氟锑酸银 $\left(\mathrm{AgSbF}_{6}\right)$ 的共催化下, 或者 在氯化金 $\left(\mathrm{AuCl}_{3}\right)$ 的催化下, 都能实现其 $\mathrm{C}-\mathrm{H}$ 键直接三 氟甲硫基化(Scheme 15).

2013 年, 吕龙和沈其龙课题组 ${ }^{[37]}$ 在 Togni 试剂的启 发下，试图合成高价碘的亲电三氟甲硫基化试剂，制备 了一种新型的亲电三氟甲硫基化试剂. 之后 Buchwald 


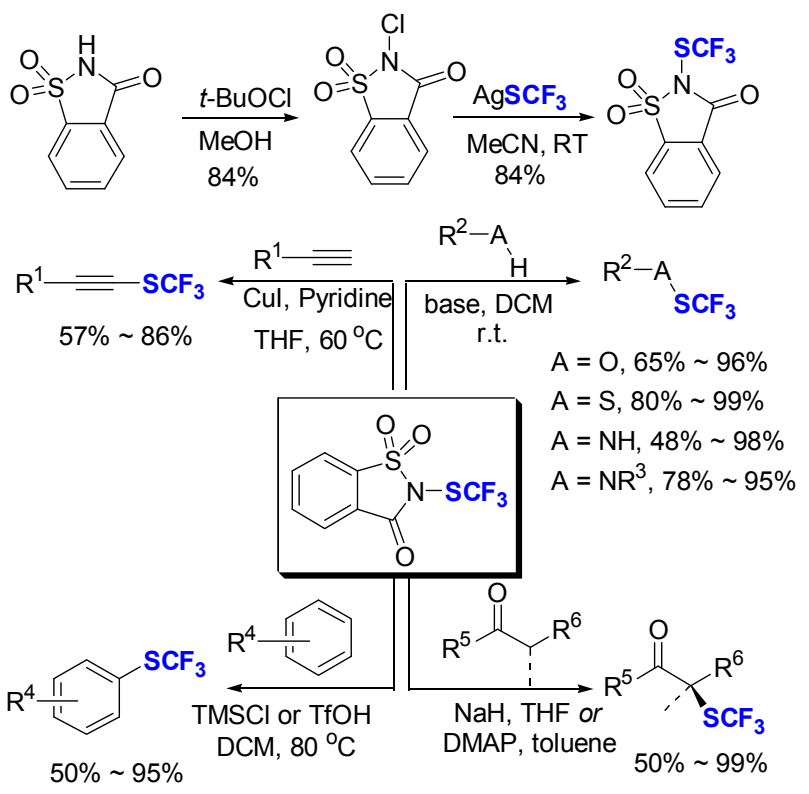

图式 $14 \mathrm{~N}$-三氟甲硫基糖精的合成及其与各种亲核体之间的 反应

Scheme 14 Synthesis of $N$-trifluoromethylthiosaccharin and its reactions with various nucleophilic substrates

课题组 ${ }^{[38]}$ 经过一系列的衍生化实验, 最终通过 $X$ 射线单 晶衍射确认了该试剂的结构, 其三氟甲硫基并不在碘原 子上, 而是在氧原子上. 首先, 沈其龙课题组 ${ }^{[37]}$ 实现了 该试剂对 $\beta$-酮酯、末端炔烃、芳基或乙烯基硼酸等亲核 类化合物的三氟甲硫基化(Scheme 16). 后来该课题组 对该试剂的应用做了进一步的拓展, 先后报道了该试剂

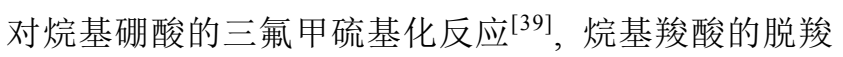
三氟甲硫基化反应 ${ }^{[40 \mathrm{a}]}$ 和吲哚衍生物的 3-位直接三氟甲

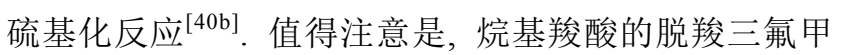

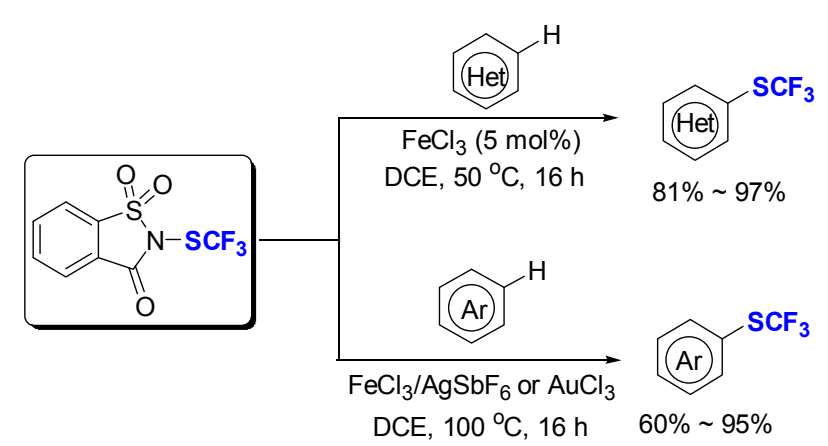

图式 15 路易斯酸催化的 $N$-三氟甲硫基糖精与(杂环)芳烃的 亲电三氟甲硫基化反应

Scheme 15 Lewis acid-catalyzed electrophilic trifluoromethylthiolation of (hetero)arenes with $N$-trifluoromethylthio-saccharin

硫基化反应可能涉及的机理是烷基羧酸脱羧产生烷基 自由基然后被亲电的三氟甲硫基所进攻得到相应的产 物.

同时，该课题组 ${ }^{[41]}$ 还用该试剂分别对 $\beta$-酮酯和吲哚 酮的不对称三氟甲硫基化做了深入的研究，在以奎宁作 手性路易斯碱时，对五元环 $\beta$-酮酯的三氟甲硫基化的对 映选择性可达 $94 \%$ ee, 对吲哚酮的对映选择性可达 $99 \%$ $e e$. 但是对于六元环或七元环的 $\beta$-酮酯底物, 该条件下 的反应转化率很低, 经过对手性相转移催化剂的篎选, 作者发现基于奎宁结构的 $N$ - 苄基奎宁溴化物 ( $N$-benzylquininium bromide) 能很好地实现这类底物的 转化, 对映选择性也较好. 2014 年, Gade 等 ${ }^{[42]}$ 利用该试 剂在铜-Boxmi 络合物催化下，也顺利地完成了 $\beta$-酮酯 的不对称三氟甲硫基化. 与上述体系不同的是，在该条 件下，对于五元环和六元环的 $\beta$-酮酯的不对称三氟甲硫

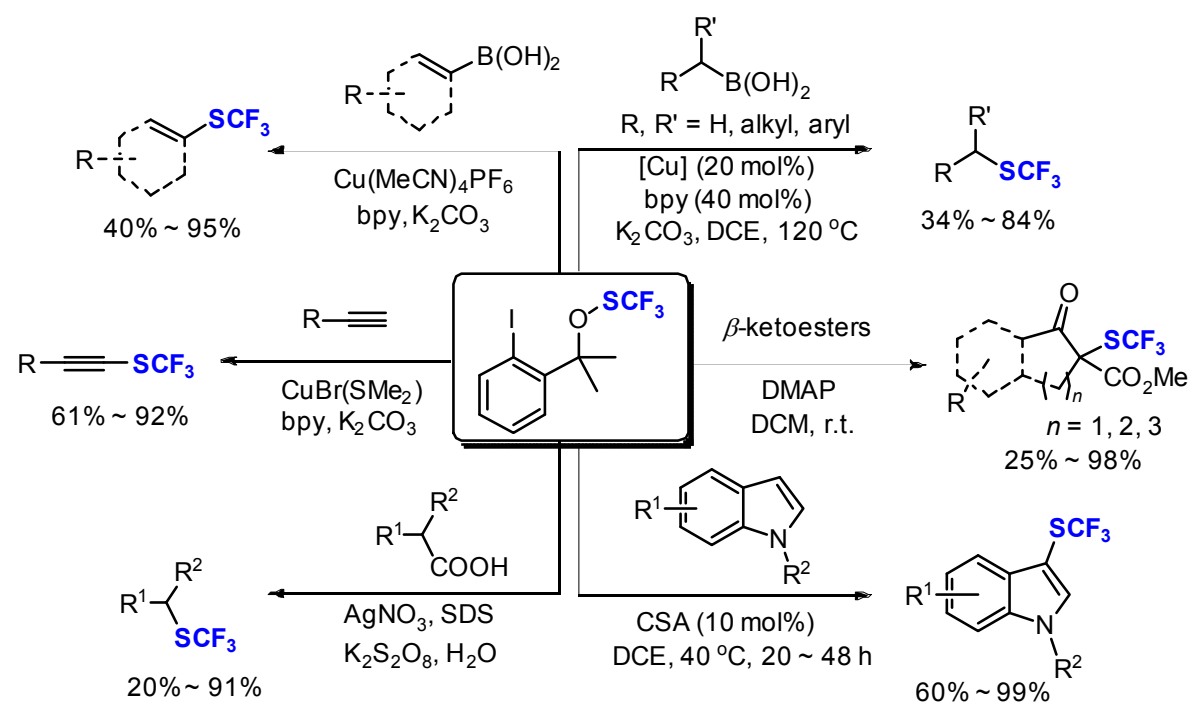

图式 16 三氟甲基取代的硫代过氧化物与嗍酸、炔烃、 $\beta$-酮酯、脂肪族羧酸及吲哚衍生物之间的三氟甲硫基化反应 Scheme 16 Trifluoromethylthiolation of boronic acids, alkynes, $\beta$-keto esters, aliphatic carboxylic acids and indoles with the trifluoromethyl-substituted thioperoxide reagent 
基化都能保持很高的收率和对映选择性(Scheme 17).

2013 年, Shibata 课题组 ${ }^{[43]}$ 报道了另一种新型的三 氟甲磺酰基高价碘叶立德试剂。该试剂在催化量的 $\mathrm{CuCl}$ 作用下，该试剂能够与多种类型的亲核试剂(如烯 胺、吲哚和 $\beta$-酮酯)反应得到三氟甲基硫醚化合物. 机理 实验表明，该试剂可能先在催化量铜盐的作用下，经过 重排产生卡宾中间体后，再经过重排生成活性中间体 $\mathbf{C}$, 中间体 $\mathbf{C}$ 可能经过单电子转移过程或者直接作为亲
电的三氟甲硫基化试剂与亲核试剂反应得所要产物 (path III), 也可能是在胺的作用下生成亲电的三氟甲硫 基取代的铵盐(path IV), 然后与各种亲核试剂反应得到 相应的三氟甲硫基化产物(Scheme 18).

在亲电的直接三氟甲硫基化反应中，一类比较特殊 的反应是通过亲核三氟甲硫基化试剂现场产生亲电的 三氟甲硫基化物种后参与反应(Scheme 19).

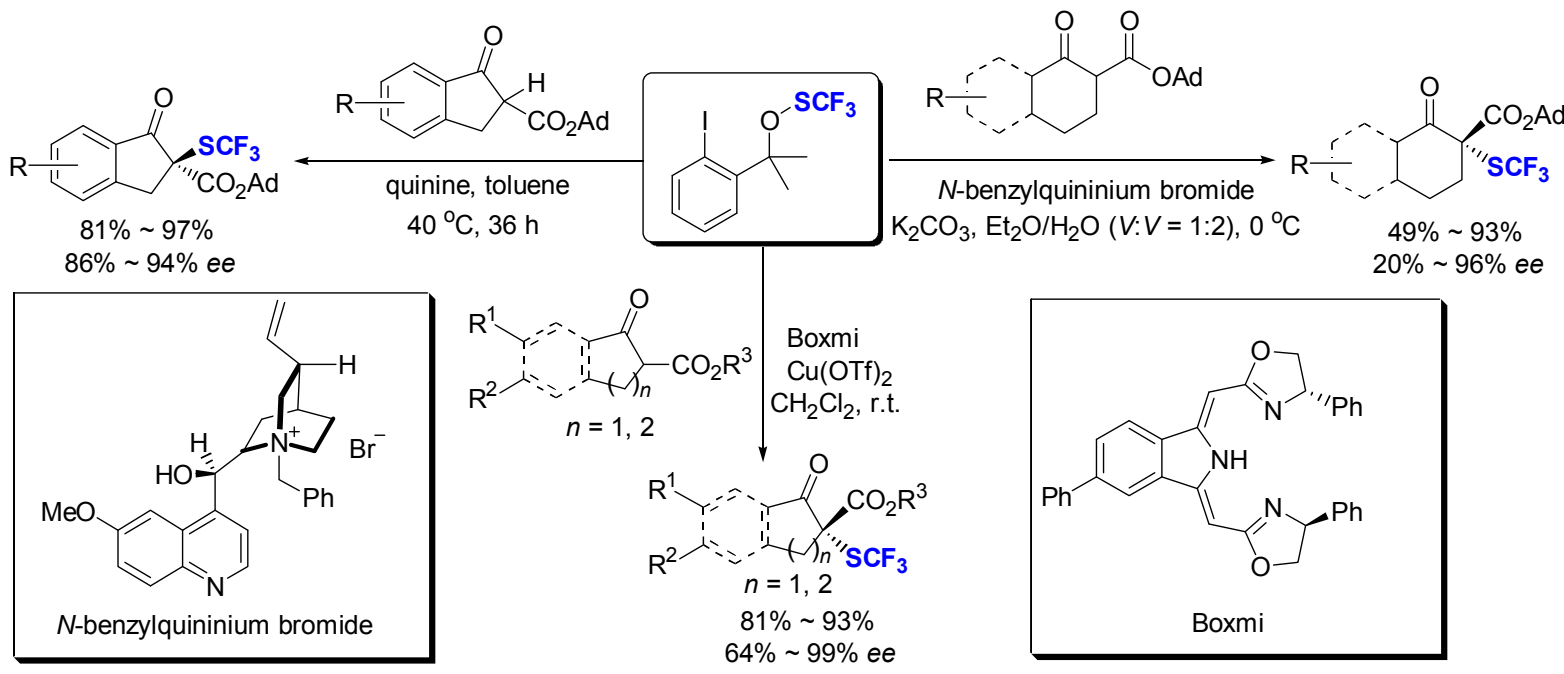

图式 17 三氟甲基取代的硫代过氧化物与 $\beta$-酮酯的不对称三氟甲硫基化反应

Scheme 17 Asymmetric trifluoromethylthiolation of $\beta$-keto esters with the trifluoromethyl-substituted thioperoxide reagent
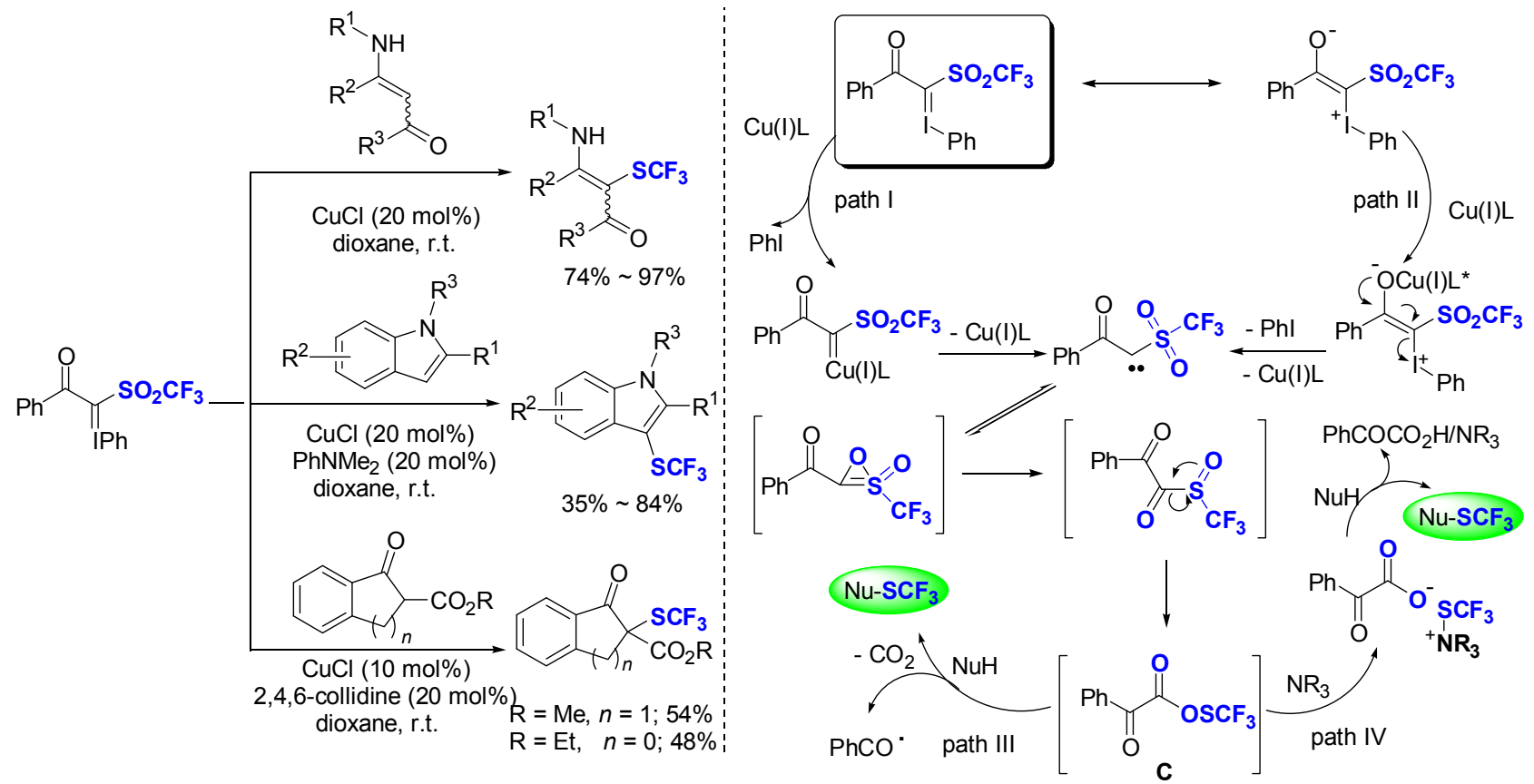

图式 18 铜催化的三氟甲磺酰基高价碘叶立德试剂与烯胺、吲哚和 $\beta$-酮酯的三氟甲硫基化反应及其可能机理

Scheme 18 Proposed mechanism for $\mathrm{Cu}(\mathrm{I})$-catalyzed trifluoromethylthiolation of enamines, indoles, and $\beta$-keto esters with trifluoromethanesulfonyl hypervalent iodonium ylide 


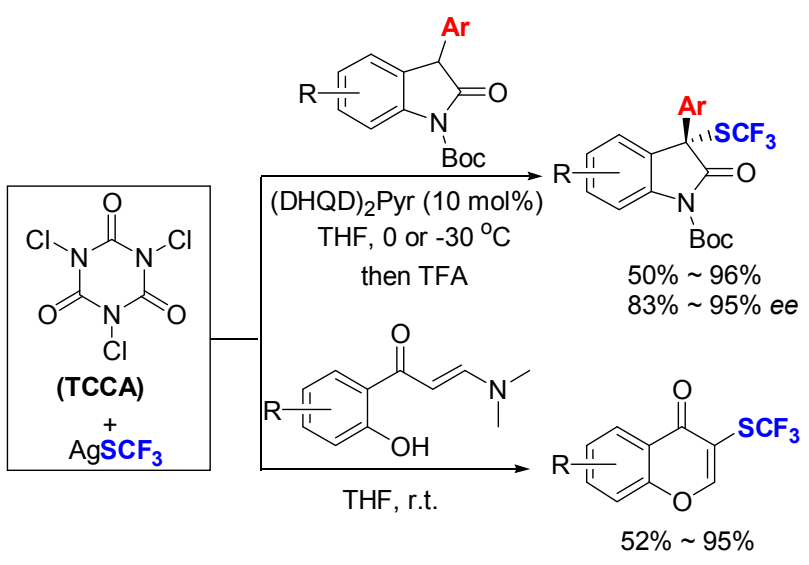

图式 19 现场生产亲电的三氟甲硫基化物种参与的三氟甲硫 基化反应

Scheme 19 Trifluoromethylthiolation via in situ generation of electrophilic trifluoromethylthio species

2014 年，南方科技大学的刘心元和谭斌等 ${ }^{[44]}$ 用 $\mathrm{AgSCF}_{3}$ 和三氯异氰尿酸(TCCA)现场生产亲电的三氟甲 硫基活性物种, 在金鸡纳碱手性催化剂(DHQD) ${ }_{2} \mathrm{Pyr}$ 的 催化下，实现对吲哚酮的不对称三氟甲硫基化，对应选 择性良好到优秀. 这丰富和补充了亲电三氟甲硫基化试 剂的不对称三氟甲硫基化反应. 中国科学院上海药物研 究所的杨春皓课题组 ${ }^{[45]}$ 也同样用这种体系合成了一系 列的三氟甲硫基取代的乙色唑衍生物. 该反应的特点在 于首先利用分子内的迈克尔加成形成烯醇中间体, 随后 与三氟甲硫基正离子反应，最后脱去二甲基胺完成相应 的转化. 利用该体系的优势在于现场生成的亲电三氟甲 硫基物种与上述亲电三氟甲硫基化试剂相比具备较高 的反应活性, 不需要添加路易斯酸便可以释放出三氟甲 硫正离子.

\section{3 亲核三氟甲硫基化}

亲核型的三氟甲硫基化试剂主要包含金属类与非 金属类两种. 在金属类三氟甲硫基试剂中应用最早的是 三氟甲硫基录试剂 $\left[\mathrm{Hg}\left(\mathrm{SCF}_{3}\right)_{2}\right]$, 能对芳基卤化物或烷基 卤化物发生亲核取代反应 ${ }^{[46]}$. 但是该试剂具有很高的 毒性和腐蚀性，限制了其应用.

三氟甲硫铯 $\left(\mathrm{CsSCF}_{3}\right)$ 也能完成对芳基卤化物或烷 基卤化物的亲核三氟甲硫基化 ${ }^{[47]} .2014$ 年, 中国科学院 上海有机化学研究所的游书力课题组 ${ }^{[48]}$ 利用该试剂, 完成了钉催化下的选择性烯丙位三氟甲硫基化. 需要指 出的是, 底物烯烃的烯丙位需要甲基碳酸酯来作为离去 基团, 产物以形成直链型烯丙基三氟甲硫醚化合物为 主. 机理实验表明, 该反应经历两次三氟甲硫基化: 首 先甲基碳酸酯作为离去基团被亲电的三氟甲硫基所取 代, 形成支链取代的三氟甲硫基化产物; 随后在钉的催
化下进一步转化为热力学更稳定的直线型三氟甲硫基 化产物(Scheme 20). 而 $\mathrm{CsSCF}_{3}$ 由于其稳定性差, 不易 制备，因而限制了该试剂的应用.

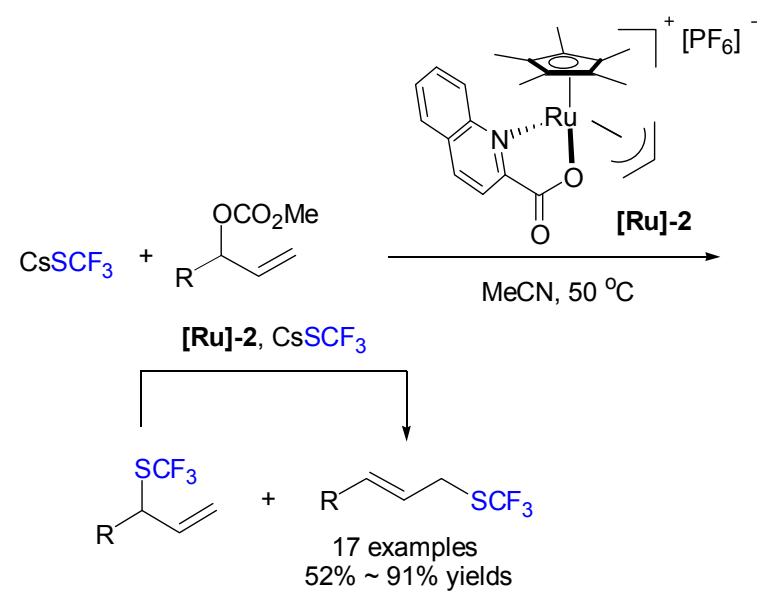

图式 20 钉催化的区域选择性烯丙位三氟甲硫基化反应 Scheme 20 Ruthenium-catalyzed regioselective allylic tri- fluoromethylthiolation reaction

相比前两种试剂，三氟甲硫化铜 $(\mathrm{I})\left(\mathrm{CuSCF}_{3}\right)$ 和三氟 甲硫银 $\left(\mathrm{AgSCF}_{3}\right)$ 的应用则广泛的多. $\mathrm{CuSCF}_{3}$ 能与传统 的亲电试剂反应，包括卤代烷烃 ${ }^{[49]}$ 、烯丙基溴 ${ }^{[50]}$ 或炔丙 基溴 ${ }^{[51]}$ 、芳基重氮 ${ }^{\text {盐 }}{ }^{[52]}$ 以及芳基卤代物 ${ }^{[53]} .2013$ 年, Rueping 课题组 ${ }^{[54]}$ 还利用 $\mathrm{CuSCF}_{3}$ 完成了对烯基碘化物 的三氟甲硫基化反应. 该反应也可使用三氟甲硫四甲基 胺盐与催化量的 $\mathrm{CuI}$ 代替三氟甲硫铜试剂(Scheme 21).

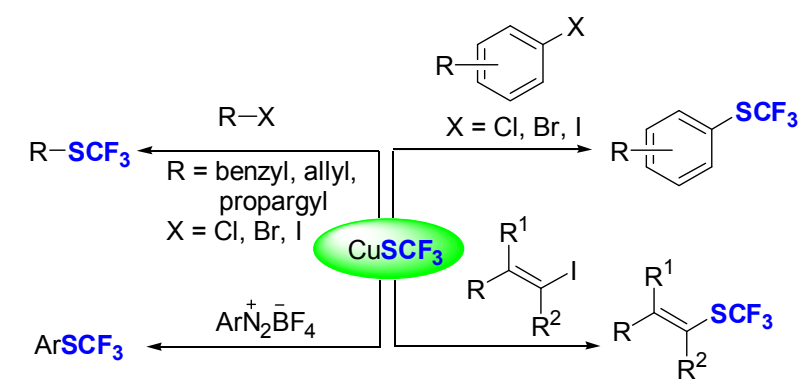

图式 21 三氟甲硫铜(I)与芳基/苄基/烯丙基卤化物、炔丙基溴 化物、乙烯基碘化物及四氟化嗍芳基重氮盐的亲核三氟甲硫 基化反应

Scheme 21 Nucleophilic trifluoromethylthiolation of aryl/benzyl/allyl halides, propargyl bromides, vinyl iodides and aryl dizaonium tetrafluororates with $\mathrm{CuSCF}_{3}$

但是 $\mathrm{CuSCF}_{3}$ 在空气中容易变质, 而且制备比较困 难, 为了克服这些缺点, 以往的方式是通过现场生成 $\mathrm{CuSCF}_{3}$ 试剂来参与反应. 2013 年, 福州大学的翁志强课 题组 ${ }^{[55]}$ 利用 2-2'联吡啶(bpy)作为配体，与 $\mathrm{CuSCF}_{3}$ 配位 后，成功制备出了空气中稳定的 2-2'联吡啶三氟甲硫铜 金属络合物 $\left[(\mathrm{bpy}) \mathrm{Cu}\left(\mathrm{SCF}_{3}\right)\right]$, 并对该络合物的反应活性 进行了考察. 芳基卤化物 ${ }^{[5]}$ 、茮溴 ${ }^{[56]}$ 、烯丙基溴化物 ${ }^{[57]}$ 
以及烷基卤代物 ${ }^{[58]}$ 都能顺利地转化(Scheme 22). 同时, 该课题也尝试用三苯基膦 $\left(\mathrm{PPh}_{3}\right)$ 代替 2,2'-联吡啶也顺利 得到了三苯基膦三氟甲硫铜金属络合物 $\left[\left(\mathrm{PPh}_{3}\right)_{2} \mathrm{Cu}-\right.$ $\left(\mathrm{SCF}_{3}\right)$ ]. 在乙腈中, $70{ }^{\circ} \mathrm{C}$ 下也能顺利完成对烯丙基溴 化物的三氟甲硫基化 ${ }^{[59]}$, 而利用硫粉与 $\mathrm{CF}_{3} \mathrm{TMS}$ 和催化 量的铜盐, 也能顺利完成对烯丙基溴化物 ${ }^{[60]}$ 和 $\alpha$-溴代 苯乙酮衍生物 ${ }^{[61]}$ 的三氟甲硫基化.

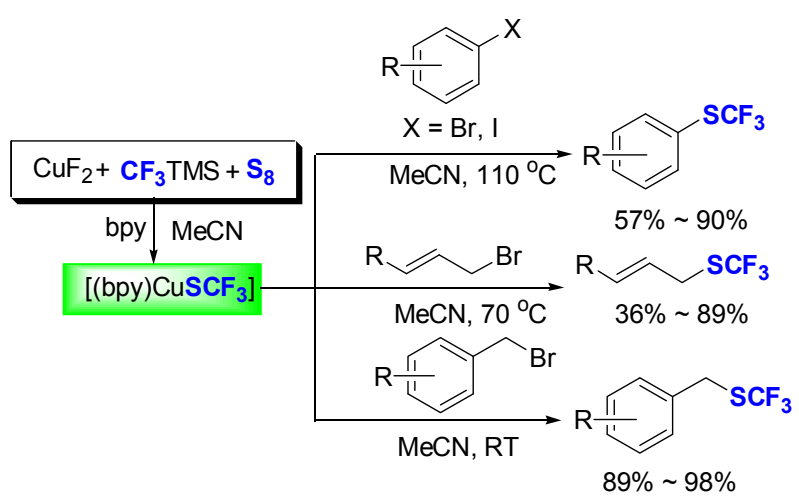

图式 22 2,2'-联吡啶三氟甲硫铜金属络合物与芳基/苄基/烯 丙基卤化物的亲核三氟甲硫基化反应

Scheme 22 Nucleophilic trifluoromethylthiolation of aryl/ benzyl/allyl halides by complex [(bpy) $\left.\mathrm{CuSCF}_{3}\right]$

从这些反应不难看出, 对于亲电性底物的三氟甲硫 基化反应，基本上都局限于卤代烃和重氮盐类型. 众所 周知, 羟基广泛存在于各种天然分子中. 而羟基本身也 能作为一种离去基团参与很多反应, 而早期报道的醇羟 基的三氟甲硫基化需要两步才能完成 ${ }^{[62]}$.

2014 年, Rueping 课题组 ${ }^{[63]}$ 用 $\mathrm{CuSCF}_{3}$ 作为亲核试 剂, 完成了醇羟基到三氟甲硫基的一步转化 (Scheme 23). 但是该体系需要加入路易斯酸活化羟基, 因此官 能团容忍性较差, 并且底物受限于烯丙基醇与苄醇类底 物.

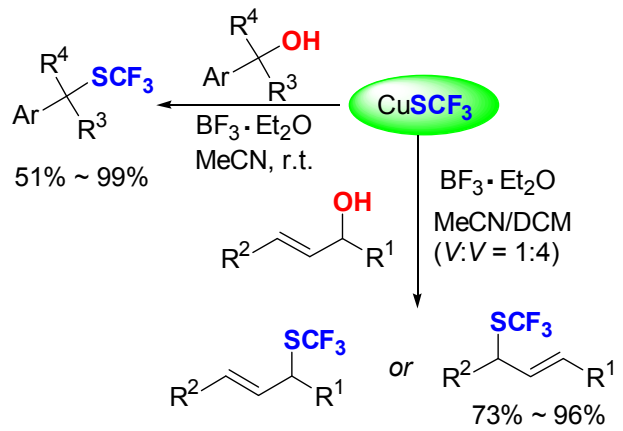

图式 23 路易斯酸活化的烯丙基醇和茮醇的直接三氟甲硫基 化反应

Scheme 23 Lewis acid-activated direct trifluoromethylthiolation of allylic and benzylic alcohols with $\mathrm{CuSCF}_{3}$

几乎与此同时, 我们课题组 ${ }^{[64]}$ 利用 $\mathrm{AgSCF}_{3}$ /四丁基
碘化铵(TBAI)体系，也顺利地实现了对醇羟基的一步三 氟甲硫基化。该体系比较简单，不需要路易斯酸活化醇 羟基，因此该反应不再局限于对酸敏感的底物，并且对 伯醇和仲醇都有良好的普适性. 该反应的机理与酸活化 着基的机理完全不同. 首先, $\mathrm{AgSCF}_{3}$ 在 TBAI 的活化下 释放三氟甲硫负离子，三氟甲硫负离子与硫代氟光气和 氟负离子存在动态平衡, 它所释放出的硫代氟光气易与 醇羟基反应生成硫代酰氟中间体，该中间体活性极高， 容易和亲核的三氟甲硫负离子发生取代反应而生成相 应的产物(Scheme 24).

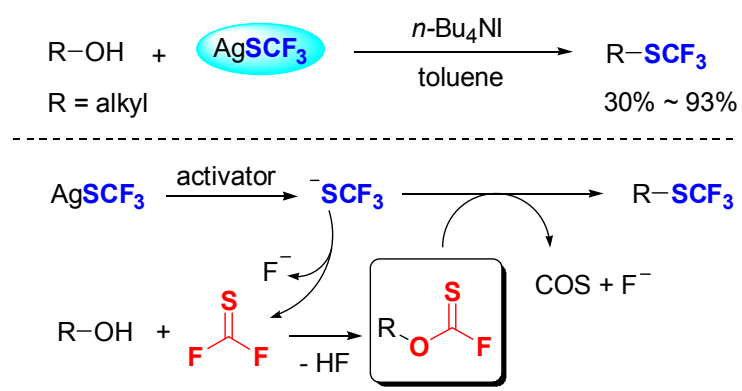

图式 24 三氟甲硫银/四丁基碘化铵体系下醇的直接脱羟基三 氟甲硫基化反应

Scheme 24 Direct dehydroxytrifluoromethylthiolation of alcohols in $\mathrm{AgSCF}_{3} / \mathrm{TBAI}$ system

尽管 $\mathrm{AgSCF}_{3}$ 的制备成本相对较高，但是它制备容 易，稳定无毒，因此在亲核的三氟甲硫基化反应中也有 着十分广泛的应用. 众所周知, $\mathrm{AgSCF}_{3}$ 易与卤代烷烃反 应，但是不易与卤代芳烃反应. 直到 2000 年, Clark 等 ${ }^{[65]}$ 发现向体系中加入 $\mathrm{KI}$ 或者 $\mathrm{TBAI}$, 能使 $\mathrm{AgSCF}_{3}$ 顺利与 亲电的卤代芳烃发生反应。尽管，该反应的机理目前依 然没有明确，但是，体系中生成 $\left[\mathrm{Ag}\left(\mathrm{SCF}_{3}\right) \mathrm{I}\right]$ 一这一活性 物种的观点被普遍认同. 其亲核能力与 $\mathrm{AgSCF}_{3}$ 相比有 所增强，因此上述机理中的三氟甲硫基负离子也很有可 能是来自这一活性物种.

近年来，三氟甲硫基化一环化反应的报道逐渐增多. 这些反应不再局限于对传统亲核底物亲电进攻，而是通 过捕捉反应中产生的缺电子的活性中间体，仅作为串联 反应中的一步而完成相应的转化. 2013 年, Lee 等 ${ }^{[66]}$ 报 道了 $\mathrm{AgSCF}_{3}$ 与三炔的反应. 作者认为 $\mathrm{AgSCF}_{3}$ 中的银 能活化炔烃从而形成芳炔活性中间体，该中间体容易被 三氟甲硫负离子进攻而完成相关转化(Eq. 3).

2014 年, 丁秋平和吴劼等 ${ }^{[67]}$ 首次报道了在银(I)催 化下从 2-炔基苯甲醛肟出发合成 1-三氟甲硫基异喹啉 类化合物的反应. 该反应可能涉及的机理也是三氟甲硫 负离子进攻缺电子的烯胺活性中间体(Eq. 4). 


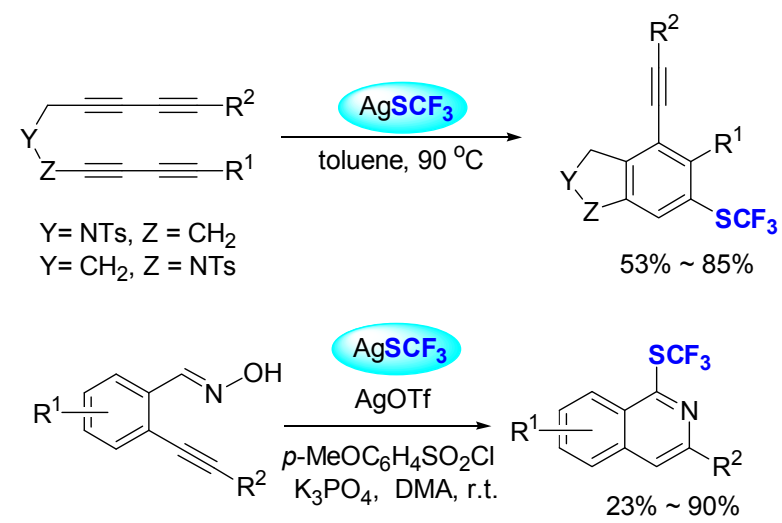

众所周知, $\alpha$-重氮甲酸酯作为碳卡宾前体, 易与各 种亲核体反应. $\alpha$-重氮甲酸酯与 $\mathrm{AgSCF}_{3}$ 的反应几乎同 时被中国科学院上海有机化学研究所的胡金波课题 组 ${ }^{[68]}$ 和北京大学的王剑波课题组 ${ }^{[69]}$ 报道. 在该反应中 都需要加入当量的铜 $(\mathrm{I})$ 盐, 铜(I)盐与 $\mathrm{AgSCF}_{3}$ 反应原位 生成 $\mathrm{CuSCF}_{3}$, 然后进攻 $\alpha$-重氮甲酸酯形成卡宾铜中间 体，经迁移插入后质子化得到相应的三氟甲硫基化产 物. 几乎与此同时, Rueping 课题组 ${ }^{[70]}$ 也直接从 $\mathrm{CuSCF}_{3}$ 出发, 实现了 $\alpha$-重氮甲酸酯的氢化三氟甲硫基化反应 (Scheme 25).

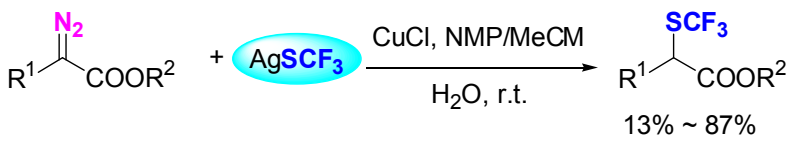

$$
\begin{aligned}
& \mathrm{R}^{1} \stackrel{\mathrm{N}_{2}}{\mathrm{COOR}^{2}}+\mathrm{AgSCF}_{3} \frac{\mathrm{Cul,} \mathrm{H}_{2} \mathrm{O}(1.0 \text { equiv. })}{\mathrm{MeCN},-25^{\circ} \mathrm{C} \text { to r.t. }} \mathrm{R}^{1} \overbrace{\mathrm{COOR}^{2}}^{\mathrm{SCF}_{3}} \\
& 34 \% \sim 88 \% \\
& \mathrm{R}^{1} \stackrel{\mathrm{N}_{2}}{\mathrm{COOR}^{2}}+\mathrm{CuSCF}_{3} \frac{\mathrm{MeCN}, 0^{\circ} \mathrm{C}, 3 \mathrm{~h}}{\mathrm{H}_{2} \mathrm{O}, \text { r.t., } 3 \mathrm{~h}} \mathrm{R}_{\substack{1 \\
51 \% \sim 86 \%}}^{\mathrm{SCF}_{\mathrm{COOR}^{2}}}
\end{aligned}
$$

图式 25 铜参与的 $\alpha$-重氮甲酸酯的三氟甲硫基化反应 Scheme 25 Copper-mediated trifluoromethylthiolation of $\alpha$-diazoesters

近年来，过渡金属催化的偶联反应一直受到广泛的 关注, 尤其是利用过渡金属催化方法向有机分子中引入 各种含氟官能团更是极大地推动了有机氟化学的发 展 ${ }^{[71]}$. 早在 2011 年, Buchwald 课题组 ${ }^{[72]}$ 报道了在大位 阻 BrettPhos 的配位下钯催化的 $\mathrm{AgSCF}_{3}$ 与芳基溴代物的 偶联反应. 在该体系中, 芳基与杂环芳基溴化物都有非 常好的普适性, 产率也十分优秀(Eq. 5). 有趣的是在该 反应中三氟甲硫基对钯催化剂并没有产生毒化作用, 对 后期的钯催化的三氟甲硫基化提供了理论基础 ${ }^{[73]}$.

2014 年, 中国科学院上海有机化学研究所的刘国 生课题组 ${ }^{[74]}$ 也使用 $\mathrm{AgSCF}_{3}$ 实现了铜催化条件下导向基 团诱导的芳基卤代物的三氟甲硫基化反应. 在催化
$\mathrm{AgSCF}_{3}$

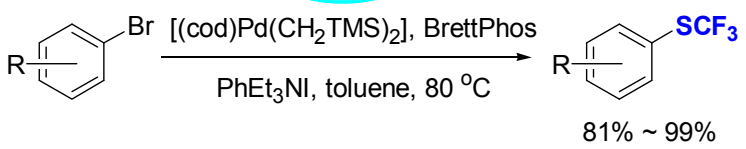

量的溴化亚铜和邻菲罗啉作用下，作者考察了各种导向 基团，如吡啶基、甲酯基、酰胺、亚胺和肟等对该反应 的影响. 他们发现当使用强配位的吡啶基为导向基团 时, 即使是芳基溴代物也能在室温与 $\mathrm{AgSCF}_{3}$ 发生取代 反应. 而对于芳基碘代物，只要将溶剂变成 DMF，即便 使用甲酯基、酰胺等弱配位的导向基团，也都能在室温 下高产率的得到三氟甲硫基化产物(Eq. 6).

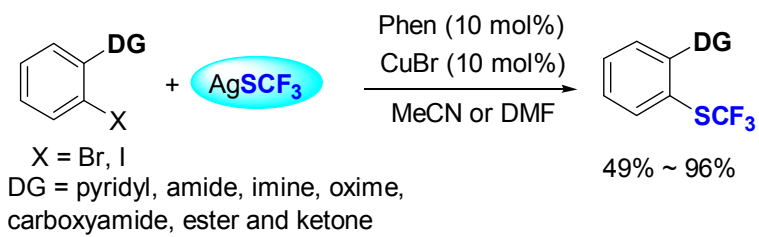

2012 年, Vicic 课题组 ${ }^{[75]}$ 报道了镍催化下的三氟甲 硫基四甲基铵盐与芳基的偶联反应. 但是该反应的普适 性不尽如人意，对强吸电子的底物并不适用，对芳基溴 化物的产率中等, 而芳基氯化物不能参与反应(Eq. 7).

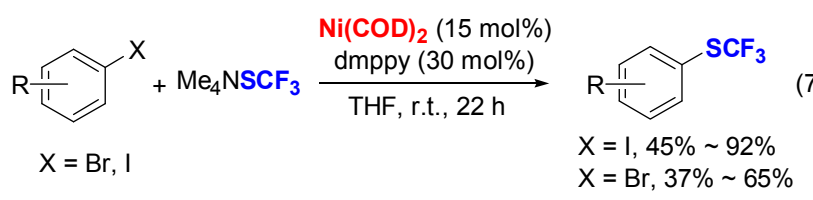

与三氟甲硫基四甲基铵盐类似的其他非金属类的 亲电三氟甲硫基化试剂的代表还包括四(二甲基胺基)乙 烯三氟甲硫盐 $\left[(\mathrm{TDAE})\left(\mathrm{SCF}_{3}\right)_{2}\right]$. 在早期的报道中，它们 也能与一些活化的芳基卤化物和卤化茮衍生物的亲核 反应 ${ }^{[76]}$. 近年来，这类试剂的应用相对较少，与金属类 的三氟甲硫基化试剂相比优势在于制备成本相对较低, 而缺点则是稳定性稍差, 一般需要在手套箱保存. 而另 一个比较特殊的非金属类的亲电型的三氟甲硫基化试 剂是 $O$-十八烷基- $S$-三氟甲基碳酸酯 ${ }^{[77]}$, 在 2003 年由 Z ard 和 Li 课题组所报道, 在氟化钾和吡咯的存在下, 它 对芦竹碱和 $\alpha$-溴取代的酮类化合物表现出很好的反应 活性(Scheme 26). 与上述两种试剂相比, 该试剂比较稳 定，原材料相对便宜，不足之处在于制备步奏稍长，并 且有关该试剂的应用报道目前还比较少.

\section{4 氧化三氟甲硫基化}

早在 1869 年, Glaser ${ }^{[78 a]}$ 就发现了在铜盐、碱以及氧 气的存在下, 苯乙炔能发生自身偶联反应, 后经过 Hay 


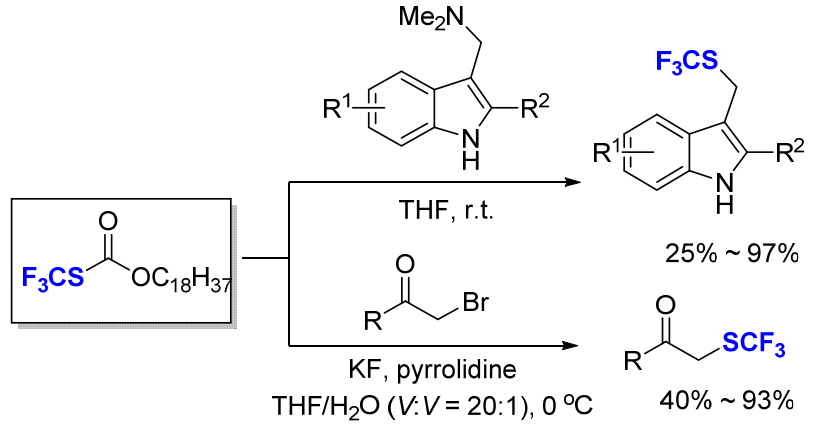

图式 $26 O$-十八烷基-S-三氟甲基碳酸酯对芦竹碱和 $\alpha$-溴取代 的酮类化合物的三氟甲硫基化反应

Scheme 26 Trifluoromethylthiolation of gramines and $\beta$-bromoketones with $O$-Octadecyl-S-trifluorothiolcarbonate

等 ${ }^{\left[{ }^{78 b}, 78 \mathrm{c}\right]}$ 的发展，使得该反应广泛应用到有机合成中， 后来被称为 Glaser-Hay 偶联反应. 1998 年, Chan, Evans 和 Lam 等 ${ }^{[79]}$ 先后发展了铜参与的芳基硼酸与醇、胺等 亲核试剂之间的偶联反应, 该反应能高效构建碳一氧键 和碳-氮键, 后来被称之为 Chan-Lam 偶联反应. 这两个 为大家所熟知的人名反应均是亲核试剂在金属和氧化 剂的作用下发生的偶联反应, 后来将这种类型的反应称 之为氧化偶联(oxidative coupling). 随着过渡金属参与 的亲核试剂之间的的氧化偶联以及过渡金属参与的 C$\mathrm{H}$ 键直接活化的偶联反应越来越受到国内外化学家的 关注，在这个大背景下，我们课题组开始尝试利用氧化 偶联的方法向有机分子中引入各种含氟官能团. 在 2010 年, 我们课题组提出了 “氧化三氟甲基化” 的新思 想, 并取得了一系列的氧化三氟甲基化研究成果 ${ }^{[80]}$.

在氧化三氟甲基化的基础上, 2012 年, 我们课题组 又发展了铜催化下芳基嗍酸的氧化三氟甲硫基化反 应 $^{[81]}$. 这种通过三组分之间的一步反应来构建碳一三氟 甲硫键丰富了三氟甲硫基化反应的发展. 该方法条件温 和, 操作简单, 并具有良好的官能团容忍性, 对于拉电 子和供电子的芳基硼酸, 都有良好的普适性(Eq. 8). 值 得说明的是, 虽然该体系采用单质硫和 $\mathrm{CF}_{3} \mathrm{TMS}$ 来提供 三氟甲硫基源, 但是机理实验却表明, 该反应可能是先 经过原位生成的芳基硫铜中间体, 随后在碳酸银的氧化 下与亲核的 $\mathrm{CF}_{3} \mathrm{TMS}$ 的发生偶联反应.

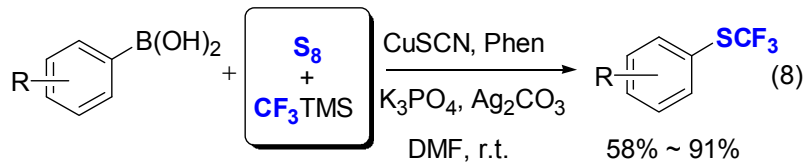

紧接着, 我们课题组 ${ }^{[82]}$ 还完成了无金属参与的端 炔氧化三氟甲硫基化反应 (Eq. 9). 有趣的是, 该反应同 样采用了单质硫和 $\mathrm{CF}_{3} \mathrm{TMS}$ 来提供三氟甲硫基源, 但是 与上面的反应不同的是, 该反应首先原位生成亲核的三
氟甲硫负离子活性物种，然后与炔烃发生偶联反应，而 且全程无金属参与. 目前, 对这一反应的机理正处于研 究阶段.

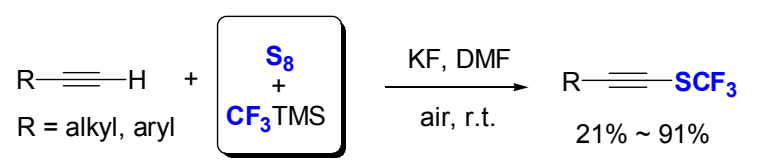

2012 年, Vicic 课题组 ${ }^{[83]}$ 发展了室温下利用三氟甲 硫四甲基铵对芳基硼酸的氧化三氟甲硫基化反应. 该体 系的优势在于在空气氛围中不需要加额外的氧化剂, 对 于乙烯基嗍酸也有良好的反应性(Eq. 10).

$$
\begin{aligned}
& \begin{array}{l}
\mathrm{R}-\mathrm{B}(\mathrm{OH})_{2}+\mathrm{Me}_{4} \mathrm{NSCF}_{3} \underset{\mathrm{C}}{\stackrel{\mathrm{Cu}(\mathrm{OTf})_{2}, \text { dtbpy }}{\longrightarrow}} \begin{array}{c}
\mathrm{Cs}_{2} \mathrm{CO}_{3}, \mathrm{THF}, \\
\text { in air, RT }
\end{array} \quad \mathrm{R}-\mathrm{SCF}_{3} \quad \text { (10) } \\
\mathrm{R}=\text { aryl, vinyl }
\end{array} \\
& \mathrm{R}=\text { vinyl, } 16 \% \sim 74 \%
\end{aligned}
$$

2013 年, 大连理工大学的李亚明等 ${ }^{[84]}$ 用三氟乙酸 钠和单质硫来提供三氟甲硫基源, 也完成了对芳基硼酸 的氧化三氟甲硫基化(Eq. 11).

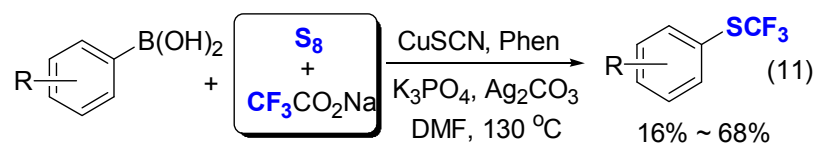

在实现了对嗍酸和末端炔烃的氧化三氟甲硫基化 反应后，我们课题组又尝试利用氧化三氟甲硫基化的思 想来实现 $\mathrm{C}-\mathrm{H}$ 键直接活化的三氟甲硫基化反应. 2014 年, 我们课题组 ${ }^{[85]}$ 完成了铜催化茮位无导向的 $\mathrm{C}\left(\mathrm{sp}^{3}\right)$ $\mathrm{H}$ 键活化的直接三氟甲硫基化反应. 以 $\mathrm{AgSCF}_{3}$ 为亲核 三氟甲硫基源，3-三氟甲基苯甲酸过氧叔丁酯为氧化剂, 可以通过一步反应直接将芐位 $\mathrm{C}-\mathrm{H}$ 键转化为 $\mathrm{C}-\mathrm{SCF}_{3}$ 键(Eq. 12).

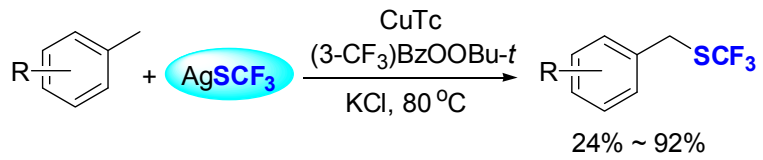

同时, 我们课题组 ${ }^{[86]}$ 还完成了铜参与的未活化末 端烯烃的氧化三氟甲硫基化反应该方法不需要预先制 备前体，并具有良好的官能团兼容性(Eq. 13).

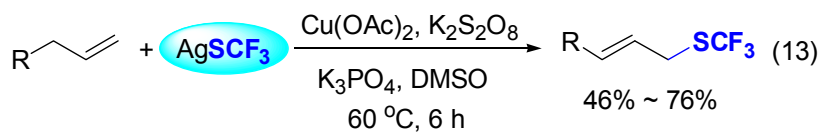

2014 年, 北京大学的黄湧课题组 ${ }^{[87]}$ 报道了导向基 团诱导的钯催化的 $\mathrm{C}-\mathrm{H}$ 键氧化三氟甲硫基化反应. 该 反应在氧化剂的作用下, 首先氧化加成形成钯(IV)中间 体，然后经过配体交换，最后还原消除得到相应的三氟 
甲硫基化产物(Eq. 14).

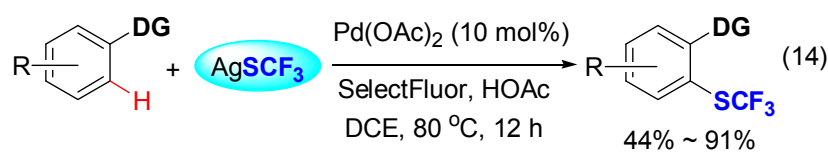

\section{5 总结与展望}

在过去的 5 年里, 三氟甲硫基化反应得到了长足的 发展. 特别是在 2013 到 2014 年, 关于三氟甲硫基化反 应的报道呈爆发式增长. 但是在三氟甲硫基化领域, 笔 者认为还有相当一部分的挑战性课题值得继续深入研 究. 首先, 目前已知的大部分三氟甲硫基化试剂在制备 成本上都很高, 发展原料易得、环境友好以及经济高效 的新型三氟甲硫基化试剂依然是研究的热点之一. 另 外, 目前的不对称三氟甲硫基化反应的底物还局限于活 泼亚甲基类化合物，例如 $\beta$-酮酯等. 而氨基酸类、胺类 以及醛酮类化合物都广泛存在于自然界中, 实现对这些 化合物的 $\mathrm{C}-\mathrm{H}$ 键活化的不对称三氟甲硫基化反应的意 义将更为深远. 分子设计与合成含三氟甲硫基的生物活 性物质将是有机氟化学的重要研究课题.

\section{References}

[1] Qing, F.-L. Chin. J. Org. Chem. 2012, 32, 815 (in Chinese). (卿凤翎, 有机化学, 2012, 32, 815.)

[2] Hansch, C.; Leo, A.; Taft, R. W. Chem. Rev. 1991, 91, 165.

[3] (a) Leroux, F.; Jeschke, P.; Schlosser, M. Chem. Rev. 2005, 105, 827.

(b) Manteau, B.; Pazenok, S.; Vors, J.-P.; Leroux, F. R. J. Fluorine Chem. 2010, 131, 140.

(c) Boiko, V. N. Beilstein J. Org. Chem. 2010, 6, 880.

(d) Meanwell, N. A. J. Med. Chem. 2011, 54, 2529.

(e) Liang, T.; Neumann, C. N.; Ritter, T. Angew. Chem., Int. Ed. 2013, 52, 8214 .

(f) He, W.-M.; Weng, Z.-Q. Prog. Chem. 2013, 25, 1071 (in Chinese).

(何伟明, 翁志强, 化学进展, 2013, 25, 1071.)

(g) Toulgoat, F.; Alazet, S.; Billard, T. Eur. J. Org. Chem. 2014,, 2415.

(h) Kalläne, S. I.; Braun, T. Angew. Chem., Int. Ed. 2014, 53, 931.

(i) Xu, X.-H.; Matsuzaki, K.; Shibata, N. Chem. Rev. 2015. 115, 731.

[4] (a) Nodiff, E. A.; Lipschutz, S.; Craig, P. N.; Gordon, M. J. Org. Chem. 1960, 25, 60.

(b) Suda, M.; Hino, C. Tetrahedron Lett. 1981, 22, 1997.

(c) Kremsner, J. M.; Rack, M.; Pilger, C.; Kappe, C. O. Tetrahedron Lett. 2009, 50, 3665.

(d) Gutmann, B.; Obermayer, D.; Reichart, B.; Prekodravac, B.; Irfan, M.; Kremsner, J. M.; Kappe, C. O. Chem. Eur. J. 2010, 16, 12182.

[5] (a) Umemoto, T.; Ishihara, S. J. Am. Chem. Soc. 1993, 115, 2156.

(b) Russell, J.; Roques, N. Tetrahedron 1998, 54, 13771.

(c) Large, S.; Roques, N.; Langlois, B. R. J. Org. Chem. 2000, 65, 8848 .

(d) Blond, G.; Billard, T.; Langlois, B. R. Tetrahedron Lett. 2001,
42, 2473.

(e) Inschauspe, D.; Sortais, J.-B.; Billard, T.; Langlois, B. R. Synlett 2003, 233.

(f) Prakash, G. K. S.; Hu, J.; Olah, G. A. Org. Lett. 2003, 5, 3253.

(g) Kieltsch, I.; Eisenberger, P.; Togni, A. Angew. Chem., Int. Ed. 2007, 46, 754 .

(h) Prakash, G. K. S.; Weber, C.; Chacko, S.; Olah, G. A. J. Comb. Chem. 2007, 9, 920.

(i) Wang, G.; Zhang, H.; Zhou, J.; Ha, C.; Pei, D.; Ding, K. Synthesis 2008, 2398 .

(j) Yagupolskii, L. M.; Matsnev, A. V.; Orlova, R. K.; Deryabkin, B. G.; Yagupolskii, Y. L. J. Fluorine Chem. 2008, 129, 131.

(k) Cherkupally, P.; Beier, P. Tetrahedron Lett. 2010, 51, 252.

(1) Riofski, M. V.; Hart, A. D.; Colby, D. A. Org. Lett. 2013, 15, 208.

(m) Kawai, H.; Yuan, Z.; Tokunaga, E.; Shibata, N. Org. Biomol. Chem. 2013, 11, 1446.

(n) Yamaguchi, K.; Sakagami, K.; Miyamoto, Y.; Jin, X.; Mizuno, N. Org. Biomol. Chem. 2014, 12, 9200.

[6] (a) Harris, J. F.; Stacey, F. W. J. Am. Chem. Soc. 1961, 83, 840.

(b) Harris, J. F. J. Am. Chem. Soc. 1962, 84, 3148.

(c) Harris, J. F. J. Org. Chem. 1966, 31, 931.

[7] Munavalli, S.; Rohrbaugh, D. K.; Rossman, D. I.; Wagner, W. G.; Durst, H. D. Phosphorus, Sulfur, Silicon Relat. Elem. 2002, 177, 1021.

[8] Haran, G.; Sharp, D. W. A. J. Chem. Soc., Perkin Trans. 1 1972, 34.

[9] Yin, F.; Wang, X.-S. Org. Lett. 2014, 16, 1128.

[10] Zhu, L.; Wang, G.; Guo, Q.; Xu, Z.; Zhang, D.; Wang, R. Org. Lett. 2014, 16, 5390.

[11] (a) Sheppard, W. A. J. Org. Chem. 1964, 29, 895.

(b) Popov, V. I.; Kondranenko, N. V.; Haas, A. Ukr. Khim. Zh. 1983, 49, 861.

(c) Rossman, D. I.; Muller, A. J.; Lewis, E. O. J. Fluorine Chem. 1991, 55, 221.

[12] (a) Andreades, S.; Harris, J. F., Jr.; Sheppard, W. A. J. Org. Chem. 1964, 29, 898.

(b) Scribner, R. M. J. Org. Chem. 1966, 31, 3671.

(c) Croft, T. S. Phosphorus Sulfur Relat. Elem. 1976, 2, 133.

[13] (a) Croft, T. S.; McBrady, J. J. J. Heterocycl. Chem. 1975, 12, 845.

(b) Haas, A.; Hellwig, V. Chem. Ber. 1976, 109, 2475.

(c) Haas, A.; Niemann, U. Chem. Ber. 1977, 110, 67.

(d) Gerstenberger, M. R. C.; Haas, A.; Liebig, F. J. Fluorine Chem. 1982, 19, 461.

(e) Gerstenberger, M. R. C.; Haas, A. J. Fluorine Chem. 1983, 23, 525 .

(f) Belanger, P. C.; Atkinson, J. G.; Rooney, C. S.; Britcher, S. F.; Remy, D. C. J. Org. Chem. 1983, 48, 3234.

[14] (a) Bayreuther, H.; Haas, A. Chem. Ber. 1973, 106, 1418.

(b) Bauer, M.; Haas, A.; Muth, H. J. Fluorine Chem. 1980, 16, 129.

(c) Popov, V. I.; Haas, A.; Lieb, M. J. Fluorine Chem. 1990, 47, 131.

[15] (a) Haas, A.; Lieb, M.; Zhang, Y. J. Fluorine Chem. 1985, $29,311$. (b) Haas, A.; Lieb, M.; Zhang, Y. J. Fluorine Chem. 1985, 30, 203.

[16] Bogdanowicz-Szwed, K.; Kawałek, B.; Lieb, M. J. Fluorine Chem. 1987, 35, 317.

[17] (a) Sharpe, T. R.; Cherkofsky, S. C.; Hewes, W. E.; Smith, D. H.; Gregory, W. A.; Haber, S. B.; Leadbetter, M. R.; Whitney, J. G. J. Med. Chem. 1985, 28, 1188.

(b) South, M. S.; Van Sant, K. A. J. Heterocycl. Chem. 1991, 28, 1017.

(c) Boese, R.; Haas, A.; Lieb, M.; Roeske, U. Chem. Ber. 1994, 
$127,449$.

[18] Tran, L. D.; Popov, I.; Daugulis, O. J. Am. Chem. Soc. 2012, 134, 18237.

[19] Ferry, A. 1.; Billard, T.; Langlois, B. R.; Bacqué, E. J. Org. Chem. 2008, 73, 9362 .

[20] Ferry, A.; Billard, T.; Langlois, B. R.; Bacqué, E. Angew. Chem., Int. Ed. 2009, 48, 8551.

[21] Alazet, S.; Zimmer, L.; Billard, T. Angew. Chem., Int. Ed. 2013, 52, 10814.

[22] Ferry, A.; Billard, T.; Bacqué, E.; Langlois, B. R. J. Fluorine Chem. 2012, 134, 160.

[23] Baert, F.; Colomb, J.; Billard, T. Angew. Chem., Int. Ed. 2012, 51, 10382.

[24] Alazet, S.; Ollivier, K.; Billard, T. Beilstein J. Org. Chem. 2013, 9, 2354.

[25] Yang, Y.; Jiang, X.; Qing, F.-L. J. Org. Chem. 2012, 77, 7538.

[26] Liu, J.; Chu, L.; Qing, F.-L. Org. Lett. 2013, 15, 894.

[27] (a) Xiao, Q.; Sheng, J.; Chen, Z.; Wu, J. Chem. Commun. 2013, 49, 8647.

(b) Sheng, J.; Li, S.; Wu, J. Chem. Commun. 2014, 50, 578.

(c) Sheng, J.; Fan, C.; Wu, J. Chem. Commun. 2014, 50, 5494.

(d) Li, Y.; Li, G.; Ding, Q. Eur. J. Org. Chem. 2014, 5017.

(e) Xiao, Q.; Zhu, H.; Li, G.; Chen, Z. Adv. Synth. Catal. 2014, 356, 3809.

[28] Alazet, S.; Zimmer, L.; Billard, T. Chem. Eur. J. 2014, 20, 8589.

[29] Alazet, S.; Zimmer, L.; Billard, T. J. Fluorine Chem. 2015, 171, 78.

[30] Munavalli, S.; Rohrbaugh, D. K.; Rossman, D. I.; Berg, F. J.; Wagner, G. W.; Durst, H. D. Synth. Commun. 2000, 30, 2847.

[31] Bootwicha, T.; Liu, X.; Pluta, R.; Atodiresei, I.; Rueping, M. Angew. Chem., Int. Ed. 2013, 52, 12856.

[32] Rueping, M.; Liu, X.; Bootwicha, T.; Pluta, R.; Merkens, C. Chem. Commun. 2014, 50, 2508.

[33] Pluta, R.; Nikolaienko, P.; Rueping, M. Angew. Chem., Int. Ed. 2014, 53, 1650.

[34] Pluta, R.; Rueping, M. Chem. Eur. J. 2014, 20, 17315.

[35] Xu, C.; Ma, B.; Shen, Q. Angew. Chem., Int. Ed. 2014, 53, 9316.

[36] Wang, Q.; Qi, Z.; Xie, F.; Li, X. Adv. Synth. Catal. 2015, 357, 355.

[37] Shao, X.; Wang, X.; Yang, T.; Lu, L.; Shen, Q. Angew. Chem., Int. Ed. 2013, 52, 3457.

[38] Vinogradova, E. V.; Muller, P.; Buchwald, S. L. Angew. Chem., Int. Ed. 2014, 53, 3125.

[39] Shao, X.; Liu, T. F.; Lu, L.; Shen, Q. Org. Lett. 2014, 16, 4738.

[40] (a) Hu, F.; Shao, X.; Zhu, D. H.; Lu, L.; Shen, Q. Angew. Chem., Int. Ed. 2014, 53, 6105 .

(b) Ma, B.; Shao, X.; Shen, Q. J. Fluorine Chem. 2015, 171, 73.

[41] (a) Wang, X.; Yang, T.; Cheng, X.; Shen, Q. Angew. Chem., Int. Ed. 2013, 52, 12860.

(b) Yang, T.; Shen, Q.; Lu, L. Chin. J. Chem. 2014, 32, 678.

[42] Deng, Q.-H.; Rettenmeier, C.; Wadepohl, H.; Gade, L. H. Chem. Eur. J. 2014, 20, 93 .

[43] Yang, Y.-D.; Azuma, A.; Tokunaga, E.; Yamasaki, M.; Shiro, M.; Shibata, N. J. Am. Chem. Soc. 2013, 135, 8782.

[44] Zhu, X.-L.; Xu, J.-H.; Cheng, D.-J.; Zhao, L.-J.; Liu, X.-Y.; Tan, B. Org. Lett. 2014, 16, 2192.

[45] Xiang, H.; Yang, C. Org. Lett. 2014, 16, 5686.

[46] (a) Man, E. H.; Coffman, D. D.; Muetterties, E. L. J. Am. Chem. Soc. 1959, 81,3575

(b) Harris, J. F. J. Org. Chem. 1967, 32, 2063.

[47] Tyrra, W.; Naumann, D.; Hoge, B.; Yagupolskii, Y. L. J. Fluorine Chem. 2003, 119, 101.

[48] Ye, K.-Y.; Zhang, X.; Dai, L.-X.; You, S.-L. J. Org. Chem. 2014, $79,12106$.
[49] (a) Munavalli, S.; Wagner, G. W.; Bashir Hashemi, A.; Rohrbaugh, D. K.; Durst, H. D. Synth. Commun. 1997, 27, 2847.

(b) Munavalli, S.; Bashir-Hashemi, A.; Rohrbaugh, D. K.; Durst, H. D. Phosphorus, Sulfur Silicon Relat. Elem. 2006, 181, 435.

[50] (a) Hanack, M.; Kühnle, A. Tetrahedron Lett. 1981, 22, 3047.

(b) Haas, A.; Hinsken, W. J. Fluorine Chem. 1985, $28,303$.

[51] (a) Hanack, M.; Massa, F. W. Tetrahedron Lett. 1981, 22, 557. (b) Haas, A.; Krächter, H.-U. Chem. Ber. 1988, 121, 1833.

[52] Adams, D. J.; Goddard, A.; Clark, J. H.; Macquarrie, D. J. Chem. Commun. 2000, 987.

[53] (a) Yagupolskii, L. M.; Kondratenko, N. V.; Sambur, V. P. Synthesis 1975, 721.

(b) Hudlicky, M. J. Fluorine Chem. 1981, 18, 383.

(c) Kondratenko, N. V.; Kolomeytsev, A. A.; Popov, V. I.; Yagupolskii, L. M. Synthesis 1985, 667.

(d) Clark, J. H.; Jones, C. W.; Kybett, A. P.; McClinton, M. A.; Miller, J. M.; Bishop, D.; Blade, R. J. J. Fluorine Chem. 1990, 48, 249.

(e) Clark, J. H.; Smith, H. J. Fluorine Chem. 1993, 61, 223.

(f) Munavalli, S.; Hassner, A.; Rossman, D. I.; Singh, S.; Rohrbaugh, D. K.; Ferguson, C. P. J. Fluorine Chem. 1995, 73, 7.

(g) Kirsch, P.; Lenges, M.; Kühne, D.; Wanczek, K.-P. Eur. J. Org. Chem. 2005, 797.

[54] Rueping, M.; Tolstoluzhsky, N.; Nikolaienko, P. Chem. Eur. J. 2013, 19, 14043.

[55] Weng, Z.; He, W.; Chen, C.; Lee, R.; Tan, D.; Lai, Z.; Kong, D.; Yuan, Y.; Huang, K.-W. Angew. Chem., Int. Ed. 2013, 52, 1548.

[56] Kong, D.; Jiang, Z.; Xin, S.; Bai, Z.; Yuan, Y.; Weng, Z. Tetrahedron 2013, 69, 6046.

[57] Tan, J.; Zhang, G.; Ou, Y.; Yuan, Y.; Weng, Z. Chin. J. Chem. 2013, 31, 921 .

[58] Lin, Q.; Chen, L.; Huang, Y.; Rong, M.; Yuan, Y.; Weng, Z. Org. Biomol. Chem. 2014, 12, 5500.

[59] Wang, Z.; Tu, Q.; Weng, Z. J. Organomet. Chem. 2014, 751, 830.

[60] Rong, M.; Li, D.; Huang, R.; Huang, Y.; Han, X.; Weng, Z. Eur. J. Org. Chem. 2014, 5010.

[61] Huang, Y.; He, X.; Lin, X.; Rong, M.; Weng, Z. Org. Lett. 2014, 16, 3284.

[62] Kolomeitsev, A. A.; Chabanenko, K. Y.; Röschenthaler, G. V.; Yagupolskii, Y. L. Synthesis 1994, 145.

[63] Nikolaienko, P.; Pluta, R.; Rueping, M. Chem. Eur. J. 2014, 20, 9867.

[64] Liu, J.-B.; Xu, X.-H.; Chen, Z.-H.; Qing, F.-L. Angew. Chem., Int. Ed. 2015, 54, 897.

[65] Adams, D. J.; Clark, J. H. J. Org. Chem. 2000, 65, 1456.

[66] Wang, K.; Yun, S. Y.; Mamidipalli, P.; Lee, D. Chem. Sci. 2013, 4, 3205.

[67] Xiao, Q.; Sheng, J.; Ding, Q.; Wu, J. Eur. J. Org. Chem. 2014, 217.

[68] Hu, M.; Rong, J.; Miao, W.; Ni, C.; Han, Y.; Hu, J. Org. Lett. 2014, $16,2030$.

[69] Wang, X.; Zhou, Y.; Ji, G.; Wu, G.; Li, M.; Zhang, Y.; Wang, J. Eur. J. Org. Chem. 2014, 3093.

[70] Lefebvre, Q.; Fava, E.; Nikolaienko, P.; Rueping, M. Chem. Commun. 2014, 50, 6617 .

[71] (a) Tomashenko, O. A.; Grushin, V. V. Chem. Rev. 2011, 111, 4475.

(b) Besset, T.; Schneider, C.; Cahard, D. Angew. Chem., Int. Ed. 2012, 51, 5048 .

(c) Furuya, T.; Kamlet, A. S.; Ritter, T. Nature 2011, 473, 470.

(d) Liang, T.; Neumann, C. N.; Ritter, T. Angew. Chem., Int. Ed. 2013, 52, 8214.

[72] Teverovskiy, G.; Surry, D. S.; Buchwald, S. L. Angew. Chem., Int 


\section{REVIEW}

Ed. 2011, 50, 7312.

[73] Xu, C.; Shen, Q. Org. Lett. 2014, 16, 2046.

[74] Xu, J.; Mu, X.; Chen, P.; Ye, J.; Liu, G. Org. Lett. 2014, 16, 3942.

[75] Zhang, C.-P.; Vicic, D. A. J. Am. Chem. Soc. 2012, 134, 183.

[76] Kolomeitsev, A.; Médebielle, M.; Kirsch, P.; Lork, E.; Röschenthaler, G.-V. J. Chem. Soc., Perkin Trans. 1 2000, 2183.

[77] Li, S. G.; Zard, S. Z. Org. Lett. 2013, 15, 5898.

[78] (a) Glaser, C. Ber. 1869, 2, 422.

(b) Hay, A. J. Org. Chem 1960, 25, 1275.

(c) Hay, A. S. J. Org. Chem 1962, 27, 3320.

[79] (a) Chan, D. M. T.; Monaco, K. L.; Wang, R.-P.; Winters, M. P. Tetrahedron Lett. 1998, 39, 2933.

(b) Evans, D. A.; Katz, J. L.; West, T. R. Tetrahedron Lett. 1998, 39, 2937. (c) Lam, P. Y. S.; Clark, C. G.; Saubern, S.; Adams, J.; Winters, M. P.; Chan, D. M. T.; Combs, A. Tetrahedron Lett. 1998, 39, 2941.

[80] Chu, L.; Qing, F.-L. Acc. Chem. Res. 2014, 47, 1513.

[81] Chen, C.; Xie, Y.; Chu, L.; Wang, R.-W.; Zhang, X.; Qing, F.-L. Angew. Chem., Int. Ed. 2012, 51, 2492.

[82] Chen, C.; Chu, L.; Qing, F.-L. J. Am. Chem. Soc. 2012, 134, 12454.

[83] Zhang, C.-P.; Vicic, D. A. Chem. Asian J. 2012, 7, 1756.

[84] Zhai, L.; Li, Y.; Yin, J.; Jin, K.; Zhang, R.; Fu, X.; Duan, C. Tetrahedron 2013, 69, 10262.

[85] Chen, C.; Xu, X.-H.; Yang, B.; Qing, F.-L. Org. Lett. 2014, 16, 3372.

[86] Zhang, K.; Liu, J.-B.; Qing, F.-L. Chem. Commun. 2014, 50, 14157.

[87] Yin, W.; Wang, Z.; Huang, Y. Adv. Synth. Catal. 2014, 356, 2998.

(Zhao, X.) 